\title{
Nonnormal del Pezzo Surfaces
}

\author{
Miles REID*
}

\begin{abstract}
This paper studies reduced, connected, Gorenstein surfaces with ample- $K$, assumed to be reducible or nonnormal. The normalisation is a union of one or more standard surfaces (scrolls and Veronese surfaces), marked with a conic as double locus. The question is how to glue these together to get a Gorenstein scheme. In characteristic 0 , the results amount to a classification of projective surfaces in the style of the $1880 \mathrm{~s}$. However, the methods involve a study of the dualising sheaf of a nonnormal variety in terms of Rosenlicht differentials, and there is a subtle pathology in characteristic $p$ due to Mori and S. Goto.
\end{abstract}

\section{§0. Introduction}

0.1 Throughout this paper, a del Pezzo surface is by definition a connected, 2-dimensional, projective $k$-scheme $X, \mathscr{O}_{X}(1)$ that is Gorenstein and anticanonically polarised; in other words, $X$ is Cohen-Macaulay, and the dualising sheaf is invertible and antiample: $\omega_{X} \cong \mathscr{O}_{X}(-1)$. For example, $X=X_{3} \subset \mathbf{P}^{3}$ an arbitrary hypersurface of degree 3 .

Under extra conditions, del Pezzo surfaces are interesting for lots of reasons: for example, as tangent cones to index 1 canonical 3-fold singularities [C3-f, 2.13]; by the "Serre correspondence", they could occur as the subschemes in $\mathbf{P}^{4}$ corresponding to sections of unstable vector bundles over $\mathbf{P}^{4}$ (the nonexistence proof of [Grauert and Schneider] has a gap). The main motivation for the present study was [Mori, Proposition 3.9], where the statement that an irreducible del Pezzo surface $X$ has $\chi\left(\mathscr{O}_{X}\right) \neq 0$ plays an essential role; I reprove here, in particular, Mori's statement that an irreducible del Pezzo surface in characteristic 0 has $\chi\left(\mathscr{O}_{X}\right)=1$ (see Corollary 4.10). This was originally proved by S. Mori and S. Goto (unpublished), and Mori was also kind enough to correct an imbecility in my proof.

0.2 I assume throughout that $X$ is reduced, but either reducible or (irreducible and) nonnormal; $\pi: Y \rightarrow X$ is the normalisation. $Y$ has $r \geq 1$ components, and is marked by an effective Weil divisor $C$, scheme-theoretically defined by the conductor ideal $I_{C, Y}=\mathscr{C}_{Y} \subset \mathscr{C}_{Y}$; as a set, $C$ is the codimension 1 double locus of $\pi$. By sub-

Communicated by K. Saito, December 21, 1990.

1991 Mathematics Subjects Classifications: 13H10, 14F10, 14J26, 14M05, 14N05

* Math. Inst., Univ. of Warwick, Coventry CV7AL, England. 
adjunction (Proposition 2.3), it's well known that the canonical Weil divisor of $Y$ is $K_{Y}=\pi^{2} K_{X}-C$, so that

$$
-K_{Y}=\pi \mathscr{C}_{X}(1)+C=(\text { ample })+(\text { effective }) .
$$

It's easy to classify the components $\mathrm{C} \subset Y$ with this property (Theorem 1.1) in terms of scrolls and $\mathbb{P}^{2}$, and in particular each connected component of $C, \mathscr{O}_{C}(1)$ must be isomorphic to a plane conic.

Write $D \subset X$ for the subscheme defined by the conductor ideal

$$
I_{D, X}=\mathscr{C}=\operatorname{Ann}\left(\pi_{\approx} \mathscr{O}_{Y} / \mathscr{O}_{X}\right) \subset \mathscr{O}_{X},
$$

and $\varphi: C \rightarrow D$ for the restriction of $\pi$. Thus $X$ is obtained by glueing together one or more components $C_{l} \subset Y_{l}$ along a morphism $\varphi: C=\amalg C_{t} \rightarrow D$. The Cohen-Macaulay or $S_{2}$ condition for $X$ is easy: $\varphi \mathscr{O}_{C} / \mathscr{O}_{D}$ must have no sections supported at points (Proposition 2.2), so that the glueing is entirely determined in codimension 1 . The combinatorics of the glueing also turns out to be straightforward (see 1.3 and Lemma 4.1); in particular if $Y$ is reducible then all the conics $C_{l}$ are isomorphic.

0.3 What makes a local ring Gorenstein? We've not heard the last of this question. The technical crux of this paper is Theorem 2.6, which characterises the predualising sheaf $\omega_{X}$ of a nonnormal scheme $X$ as the sheaf of Rosenlicht differentials, that is, rational sections of the predualising sheaf $\omega_{Y}$ of the normalisation $Y$ with poles along $C$, and whose residues along $C$ have zero trace on the generic fibres of $\varphi: C \rightarrow D$. This gives the necessary and sufficient conditions of Corollary 2.8 on $\mathrm{C} \subset Y$ and $\varphi: C \rightarrow D$ for $X$ to be Gorenstein, for example: $\omega_{Y}(C)$ is an invertible $\mathscr{O}_{Y}$-module, $\omega_{X}$ is Gorenstein in codimension 1 and $\omega_{C}$ has an $\mathscr{O}_{C}$-basis $s \in \operatorname{ker}\left\{\operatorname{Tr}_{C / D}: \varphi, \omega_{C} \rightarrow \omega_{D}\right\}$. In codimension 1, where $\pi, \mathscr{O}_{Y}$ localised at a prime divisor of $X$ is a product of DVRs, the condition for $\mathscr{O}_{X} \subset \pi \cdot \mathscr{C}_{Y}$ to be Gorenstein translates into an interesting question on subrings $\mathscr{O}_{D}$ "half-filling" a product $\varphi \cdot \sigma_{C}=\prod A_{\imath}$ of Artinian quotients of DVRs (see 3.4-5). This result may be useful in other contexts, since Gorenstein in codimension 1 is the essential prerequisite for working with conditions such as log canonical singularities on nonnormal schemes. Even in the classic case of curves over an algebraically closed field (Rosenlicht, Serre), it gives rise to lots of unsolved problems, and my elementary results Theorem 3.7 may be new even in this case.

The material on duality and Rosenlicht differentials in $\S \S 2-3$ is written up in much more generality than needed for the geometry of del Pezzo surfaces; it is part of a continuing attempt to write up Grothendieck duality in an absolutely elementary way (see also [Reid]). $\S 3$ is a laundry job on [Serre, Ch. IV, §11]. (I hope that's not lèsemajesté.) I haven't tried to find historically correct attributions for these ideas.

0.4 Working with the Gorenstein condition is easy if all the $C_{l}$ are reduced, when $X$ has ordinary double points in codimension 1 (see Theorem 3.7, (I) and 4.2-3). But in 
the nonreduced case, the question is quite subtle, and the answer depends on char $k$. In any characteristic, Sing $X=\Gamma$ is an irreducible curve of degree 1 for the polarisation, and by Theorem 3.7, (II) the transverse singularity of $X$ along the generic point of $\Gamma$ is a cusp $\left(y^{2}=x^{3}\right)$ if $r=1$, a tacnode $\left(y^{2}=x^{2} y\right)$ if $r=2$, and $r$ concurrent lines in $\mathbf{A}^{(1-1)}$ with no $(r-1)$ in a hyperplane for $r>3$ (if $r=3$, a plane ordinary triple point $x y(x-y)=0)$. If char $k=0$ then $\Gamma \cong \mathbb{P}^{1}$ and $D, \mathscr{O}_{D}(1)$ is isomorphic to a first order neighbourhood of $\mathbf{P}^{1}$ in $\mathbf{P}^{r}$, with the morphism $\varphi: \amalg C_{l} \rightarrow D$ linear on each component (see Proposition 4.9); this is essentially equivalent to the main result $\chi\left(\mathscr{O}_{X}\right)=1$ (see Corollary $4.10,(\mathrm{I})$ ). However, in characteristic $p$ the curve $\Gamma$ can have "wild" cusps (see 4.4, 4.7 and 4.11-12), essentially because the unknown subring $\mathscr{O}_{D} \subset \varphi_{*} \mathscr{O}_{C}$ is specified by a derivation (Proposition 3.9), and $\mathrm{d} x^{p} \equiv 0$. Any number of wild cusps can occur, and $\chi\left(\mathscr{G}_{X}\right)$ can be arbitrarily negative. Thus for nonnormal varieties in characteristic $p$, Gorenstein is a weaker condition than in characteristic 0 , a new kind of pathology discovered by Mori and Goto.

0.5 It follows easily that if char $k=0$ then $H^{1}\left(X, \mathscr{G}_{X}(n)\right)=0$ for all $n$, in particular $\chi\left(\mathscr{O}_{X}\right)=1$, and a general element $x_{0} \in H^{0}\left(X, \mathscr{C}_{X}(1)\right)$ is a non-zerodivisor for $\mathscr{O}_{X}$ (see Corollary 4.10,(I)); well-known arguments then show (Corollary 4.10, (II)) that $X$ has the usual projective embedding properties of nonsingular del Pezzo surfaces or curves of genus 1: $\mathscr{O}_{X}(1)$ is very ample if $\operatorname{deg} X \geq 3$, the image is ideal-theoretically an intersection of quadrics if $\operatorname{deg} X \geq 4$, and $X$ is a weighted hypersurface or complete intersection $X_{6} \subset \mathbb{P}\left(1^{2}, 2,3\right), X_{4} \subset \mathbb{P}\left(1^{3}, 2\right), X_{3} \subset \mathbb{P}^{3}$ or $X_{2,2} \subset \mathbb{P}^{4}$ if $\operatorname{deg} X \leq 4$. See 1.3-4 for examples.

However, if char $k=p$ then $H^{1}\left(X, \mathscr{O}_{X}\right)$ can be arbitrarily large; for an irreducible surface $X$ this happens only when $X$ has a curve $\Gamma$ of cusps, and $\Gamma$ itself has "wild" cusps. In particular, this cannot happen in characteristic $\geq 5$ if $X$ has only hypersurface singularities (see 4.4 and 4.7); I guess that a similar conclusion holds under other reasonable conditions, e.g., $X$ lifts to characteristic $p^{2}$.

0.6 Conjecture. Assume that char $k=0$, and that $X$ is 1-connected but not reduced; then $X, C_{X}(1)$ is projectively Cohen-Macaulay (that is, $H^{\prime}\left(X, C_{X}(n)\right)=0$ for $i=1$ and all $n$, and for $i=2$ and $n \geq 0)$, and a general element $x_{0} \in H^{0}\left(X, C_{X}(1)\right)$ is $\mathscr{O}_{X}$-regular, so all the projective embedding properties hold as above. Finding the correct notion of 1-connected in general is part of the problem; for example, if $X$ is locally a divisor in a smooth 3-fold and $X=A+B$ then $C_{A}(-B)$ is an invertible sheaf, and 1 -connected means that $\operatorname{deg}_{\gamma_{X}(1)} C_{A}(-B)<0$. By adjunction, $\omega_{A}=\omega_{X} \otimes C_{A}(-B)$, so that, as in the reduced case, this amounts to $K_{A}{ }^{-1}>$ (ample Cartier).

0.7 The great debate. Rend. del circolo matematico di Palermo 1 (1887), p. 382 records the admission to the circle of dottore Pasquale del Pezzo, marchese di Campodisola. It would be interesting to know why Corrado Segre writing in the same 
volume (p. 218, 220, 221), along with every subsequent Italian writer, spells the Marquis' name incorrectly with a capital D.

0.8 Acknowledgements. I thank S. Mori for his help, and for encouraging me to write up my proof, and M. Miyanishi whose 1981 seminars on [Mori] introduced me to the problem (and to much else besides). The initial version of this paper was intended for the proceedings of the wonderful Kinosaki conference in Dec 1981, and I apologise for the delay in writing up the talk; however, as with other products consumed at Kinosaki, the material undoubtedly improves on maturing for several years. I'm very grateful to S. Mori and K. Saito for inviting me to Japan, and to RIMS, Kyoto Univ. for employing me during 1989-1990, when this paper was written. J. Kollár and N. Shepherd-Barron helped correct an error in the adjunction formula of Theorem 2.12. Exercise 4.12 answers a question raised by Shepherd-Barron and Kollár.

\section{Contents}

$\S 1$. The normalised variety $C \subset Y$

$\S 2$. Normalisation and dualising sheaves

Appendix to $\$ 2$. Adjunction for a finite morphism

$\S 3$. What happens in codimension 1

$\S 4$. The glueing map $\varphi: C \rightarrow D$ and proof of Theorem 1.5

References

\section{$\S 1$. The Normalised Variety $C \subset Y$}

In (1.1-2), $Y$ denotes one component of the normalisation of $X$. As explained in Theorem 2.3, it follows from subadjunction that $-K_{Y}=H+C$, where $H=\mathscr{O}_{Y}(1)$ is an ample Cartier divisor and $C>0$ an effective Weil divisor. I tabulate the information on $C \subset Y$ in the following form, to enable the many readers familiar with the result to skip to the next section.

1.1 Theorem. Pairs $C \subset Y$ are listed as follows:

\begin{tabular}{|c|c|c|c|c|}
\hline Case & $Y, \mathscr{O}_{Y}(1)$ & degree $\left(\mathscr{O}_{Y}(1)\right)^{2}$ & Class of $C$ & Nature of $C$ \\
\hline (a) & $\mathbf{P}^{2}, \mathscr{O}_{\mathbf{P}^{2}}(1)$ & 1 & $\begin{array}{l}\mathscr{O}_{\mathbf{p}^{2}}(2), \text { that is, } \\
\text { plane conic }\end{array}$ & $\begin{array}{l}\text { (a1) smooth conic } \\
\text { (a2) line pair } \\
\text { (a3) double line }\end{array}$ \\
\hline (b) & $\mathbf{P}^{2}, \mathscr{O}_{\mathbf{p}^{2}}(2)$ & 4 & $\mathscr{O}_{\mathbf{p}^{2}}(1)$ & smooth conic \\
\hline (c) & $\begin{array}{l}\mathbf{F}_{a: 0}, a A \\
\quad \text { for } a \geq 2\end{array}$ & $a$ & $\begin{array}{l}2 A \text {, that is, } \\
2 \text { generators, } \\
\text { or if } a=2 \text { only: }\end{array}$ & $\begin{array}{l}\text { (c1) line pair } \\
\text { (c2) double line } \\
\text { (c0) smooth conic }\end{array}$ \\
\hline
\end{tabular}




\begin{tabular}{|l|c|c|l|l|}
\hline (d) & $\begin{array}{c}\mathbf{F}_{a ; 1},(a+1) A+B \\
\text { for } a \geq 0\end{array}$ & $a+2$ & $\begin{array}{c}A+B \\
\text { or if } a \leq 1 \text { only: }\end{array}$ & $\begin{array}{l}\text { (d1) line pair } \\
(\mathrm{d} 0) \text { smooth conic }\end{array}$ \\
\hline (e) & $\mathbf{F}_{a: 2},(a+2) A+B$ & $a+4$ & $B$ & smooth conic \\
\hline
\end{tabular}

Here $\mathbf{F}_{a}$ is the usual C. Segre-P. del Pezzo scroll [Segre, del Pezzo 1], $A$ and $B$ its fibre and negative section; and

$$
\mathbf{F}_{a: k}=\left(\mathbf{F}_{a}, \mathscr{O}((a+k) A+B)\right)
$$

is the embedded scroll $\mathbf{F}_{a ; k} \subset \mathbf{P}^{a+2 k+1}$ of degree a $+2 k$, with negative section $B$ of degree $k$, except for $k=0$, when $\mathbf{F}_{a ; 0}$ is the cone over a rational normal curve, polarised by $\mathscr{O}(1)=\mathscr{O}(a A)$. The two exceptional cases $(\mathrm{c} 0)$ and $(\mathrm{d} 0)$ with a $=0$ both correspond to a quadric of $\mathbf{P}^{3}$ with a smooth hyperplane section. Note that a double generator of a cone is isomorphic to a double line in $\mathbf{P}^{2}$, for example because it is a projective cone over a tangent vector $k[\varepsilon] /\left(\varepsilon^{2}\right)$, or because $\omega_{C} \cong \mathscr{O}_{C}(-1)$.

1.2 Proof. This theorem is presumably traditional, but Mori theory gives an amazingly clean proof. I do a minimal resolution $f: S \rightarrow Y$, set $L=f^{4} H=f^{\prime}\left(-K_{Y}-C\right)$ and $C^{\prime}=f^{\prime} C$ (birational transform); then $L$ is a nef and big Cartier divisor, having positive intersection number with every component of $C^{\prime}$. Write

$$
K_{S}+C^{\prime}=f^{*}\left(K_{Y}+C\right)-Z
$$

clearly $Z$ is a Cartier divisor supported on the exceptional locus of $f$, and $\left(K_{S}+C^{\prime}\right) \Gamma \geq 0$ for every exceptional curve $\Gamma$, hence $Z \geq 0$. Now $K_{S}+L=-C^{\prime}-Z$ is not nef, so that by the theorem on the cone [Mori, 1.4 and 2.1] it follows that $S$ has an extremal rational curve $\ell$ with $\left(K_{S}+L\right) \ell<0$. But $\ell$ cannot be a $(-1)$-curve, since a $(-1)$-curve $m$ satisfies $K_{S} m=-1, L m>0$; therefore either $S=\mathbf{P}^{2}$ and $\operatorname{deg} L \leq 2$ or $S$ is a $\mathbf{P}^{1}$-bundle and $\operatorname{deg} L \leq 1$ on the fibres. The theorem follows on sorting out the last possibility.

Q.E.D.

1.3 Reassembling the pieces. A nonnormal del Pezzo surface $X$ is obtained by glueing together a number $r \geq 1$ of the building blocks $C_{l} \subset Y_{l}$ of Theorem 1.1. How to ensure that $X$ is Gorenstein is the main theme of $\S \S 2-4$; since $\mathscr{O}_{Y}\left(-K_{Y}-C\right)=\mathscr{O}_{Y}(1)$ is invertible, Corollary 2.8 reduces this to a question on the glueing morphism $\varphi: C=\coprod C_{t} \rightarrow D$.

Here I present some classes of examples of the finished product $X$ in the spirit of the projective geometry of the 1880 s. 1.4 deals with cases specific to $\operatorname{deg} X \leq 2$, when $\mathscr{O}_{X}(1)$ is not very ample.

(A) Project $\quad \mathbf{F}_{a: 2} \subset \mathbf{P}^{a+5}$ to $\mathbf{P}^{a+4}$ from a point $P$ in the plane of the conic $B$ but not on $B$; the projection has a line $\ell$ of ordinary double points. The same construction for the Veronese surface (case (b) of the theorem) is of course the well-known Bordello surface $F_{(1)}^{4}[4] \subset \mathbf{P}^{4}$ of [Semple and Roth, p. 132], which is a complete intersection of two quadrics with a double line. 
Note that in characteristic 2, two cases occur, since all tangents to a plane conic pass through a point $Q$; the projection $\varphi: B \rightarrow \ell$ from $Q$ is inseparable, whereas the projection from any other point not on $B$ is separable.

(B) Let $C_{1} \subset Y_{1}$ be any two elements of (a1, b, c0, d0, e), not both from (a1); that is, the $C_{t}$ are smooth conics, and not both of the $Y_{l}$ are planes. Embed $Y_{1}$ and $Y_{2}$ into a common projective space $\mathbb{P}^{n}$ such that the subspaces spanned by $Y_{1}$ and $Y_{2}$ intersect in the planes of the $C_{l}$, and the $C_{l}$ are identified.

$\left(\mathrm{C}_{1}\right)$ Let $C \subset Y$ be an element of (c1) or (d1) of degree $\geq 3$, so that $C$ is a line pair spanning a plane $\Pi$ of the projective space $\mathbb{P}^{n}$ containing $Y$ with $n \geq 4$; make a linear projection $Y \rightarrow X \subset \mathbb{P}^{n-1}$ from a point $P \in \Pi \backslash C$. In case (c1), $X$ is the projective cone over a nodal rational curve of degree $\geq 3$. This case can be viewed as the degenerate case $r=1$ of the following $\left(\mathrm{C}_{1}\right)$.

$\left(\mathrm{C}_{r}\right)$ Let $C_{\imath} \subset Y_{\iota}$ be any $r \geq 2$ elements of (a2, c1, d1), not both from (a2) if $r=2$; that is, the $C_{l}$ are line pairs, and not both the $Y_{l}$ are planes if $r=2$. Embed the $Y_{l}$ into a common projective space as a cycle of surfaces meeting along lines of $C_{i}$, with a common vertex.

In other words, name the lines of the pair $C_{l}$ as $C_{l}=\ell_{l} \cup \ell_{l}^{\prime}$ (the subscripts are cyclic, so $i=r+1$ counts as $i=1$ ). In $\mathbb{P}^{n}$, choose a vertex $P$ and $r$ linearly independent lines $m_{l}$ through $P$, and embed the $Y_{l}$ so that $\ell_{l}$ is glued to $m_{l-1}$, and $\ell_{l}^{\prime}$ to $m_{l}$, with the subspaces spanned by $Y_{\imath}$ and $\coprod_{\jmath \neq 1} Y$, intersecting only in the plane of $m_{i-1}$ and $m_{i}$, so that they are as linearly independent as possible. The superfluous notation $\ell_{l}^{\prime \prime}=\ell_{t+1}$ will be convenient later, so that the two lines of $C$ lying above $m_{l}$ are $\ell_{l}^{\prime}$ and $\ell_{l}^{\prime \prime}$.

$\left(D_{1}\right)$ The projective cone over a cuspidal rational curve of degree $\geq 3$. This can be viewed as a degenerate case of the following $\left(D_{r}\right)$.

$\left(\mathrm{D}_{r}\right)$ Let $C_{1} \subset Y$, be any $r \geq 2$ elements of $(\mathrm{a} 3, \mathrm{c} 2)$, not both from (a3) if $r=2$; that is, the $C_{l}$ are double lines, and not both the $Y_{l}$ are planes if $r=2$. Embed the $Y_{\imath}$ into a common projective space meeting along a line, with a single nondegenerate linear dependence relation between the $r$ planes of $C_{l}$.

In other words, in $\mathbb{P}^{\prime}$, choose a line $\ell$ and $r$ planes $\pi_{l}$ through $\ell$ such that any $(r-1)$ of them span the same $\mathbb{P}^{r}$. Embed the $Y_{i}$ so that $C_{l}$ is identified with the double line $2 l \subset \pi_{t}$, and so that the subspaces spanned by $Y_{i}$ and $\amalg_{J \neq l} Y_{J}$ intersect in $\pi$, only.

1.4. Degree $\mathbb{1}$ and 2. I describe briefly the del Pezzo surfaces of degree 1 and 2, leaving most of the computations to the reader; a similar description of surfaces of degree 3,4 and 5 by equations is also possible, and is an interesting exercise.

Consider first degree 1 . Let $Y=\mathbb{P}^{2}$ with coordinates $u_{1}, u_{2}, u_{3}$ and $C:(q=0)$, where

$$
q=u_{2}^{2}-u_{1} u_{3}, u_{2}^{2}-u_{1}^{2} \text { or } u_{2}^{2} \text {. }
$$


It's easy to see that setting $x_{1}=u_{1}, x_{2}=u_{3}, y=q$ and $z=u_{2} q$ defines a birational morphism $\pi: Y \rightarrow X_{6} \subset \mathbb{P}\left(1^{3}, 2,3\right)$, with image $X=X_{6}$ the sextic hypersurface defined by

$$
z^{2}=y^{3}+x_{1} x_{2} y^{2}, \quad z^{2}=y^{3}+x_{1}^{2} y^{2} \quad \text { or } z^{2}=y^{3} .
$$

In characteristic $\neq 2$, the map $\varphi: C \rightarrow D=\mathbb{P}^{1}:(y=z=0)$ is the quotient by the $Z / 2$ action $u_{2} \mapsto-u_{2}$. In the second and third case, $X$ is a weighted cone over a nodal or cuspidal rational curve, defined by a polarisation of degree 1 .

In characteristic 2 , the same equations still define a normalisation, but in the first case $\varphi: C \rightarrow D$ is an inseparable cover of $\mathbf{P}^{1}$ by a nonsingular conic; a separable example is provided by the normalisation of $z^{2}+x_{1} y z=y^{3}+x_{2}^{2} y^{2}$.

It's interesting to observe that whereas for del Pezzo surfaces with isolated singularities, those of degree 1 are by far the most complicated, in the nonnormal case those of degree 1 are very few, and simple to describe.

Now for degree 2. It's obvious that if $\left(q=q_{2}\left(x_{1}, x_{2}, x_{3}\right)=0\right) \subset \mathbf{P}^{2}$ is a conic then the weighted quartic $X=X_{4}:\left(y^{2}=q^{2}\right) \subset \mathbb{P}\left(1^{3}, 2\right)$ is a del Pezzo surface of degree 2, consisting of 2 copies of $Y \cong \mathbb{P}^{2}$ glued along the conic $(q=0)$.

If $Y$ is irreducible of degree 2, then from the table of Theorem 1.1 it must be a quadric of $\mathbb{P}^{3}$, with $C$ a hyperplane section. Consider, in characteristic $\neq 2$, the plane quartics $\ell^{2} q$ with a double line $/$ and $q$ a conic in the following 5 case: a nonsingular conic not tangent to $\ell$, a nonsingular conic tangent to $\ell$, a line pair with vertex not on $\ell$, a line pair with vertex on $\ell$ but not containing $\ell$, or a line pair with $\ell$ as a component. Let $X=X_{4}:\left(y^{2}=\ell^{2} q\right) \subset \mathbb{P}\left(1^{3}, 2\right)$ be the double cover of $\mathbf{P}^{2}$ branched in $/^{2} q$. It's easy to see in the 5 case that the normalisation of $X$ is a surface $C \subset Y$ of type (d0, d1, $\mathrm{c} 0, \mathrm{c} 1, \mathrm{c} 2)$.

1.5 Main theorem. Under the "tame" assumption of 4.7, the examples of 1.3-4 provide a complete classification of nonnormal del Pezzo surfaces.

The "tame" condition covers all cases with char $k=0$, or $C$ reduced, or char $k \geq 5$ and $X$ locally a divisor in a nonsingular 3-fold; it is equivalent to $H^{1}\left(X, C_{X}\right)=0$ or $\chi\left(G_{X}\right)=1$. See 4.4 and $4.11-12$ for counterexamples in the remaining cases.

\section{\$2. Normalisation and Dualising Sheaves}

2.0 In this section $X$ is a purely $n$-dimensional variety, and $\pi: Y \rightarrow X$ its normalisation. More generally $X$ could be a reduced Noetherian scheme under the assumptions that the normalisation $\pi: Y \rightarrow X$ is finite and $Y$ has a dualising complex. The most general category for the dualising complex has never been definitively established, but, for example, if $Y$ is contained in a Gorenstein ambient scheme then dimensions and codimensions are well defined ("universally catenary"), and $Y$ has a dualising complex, whose top cohomology is the predualising sheaf $\omega_{Y}$. 
The aim is to use Grothendieck duality to describe $\omega_{Y}$ in terms of $\omega_{X}$ and vice versa. Note that all the sheaves here are coherent $\mathscr{O}_{X}$-modules.

2.1 Write $\mathscr{C}=\operatorname{Ann}\left(\pi, \mathscr{O}_{Y} / \mathscr{O}_{X}\right) \subset \mathscr{O}_{X}$ and $\mathscr{C}_{Y}=\mathscr{C} \cdot \mathscr{O}_{Y} \subset \mathscr{O}_{Y}$ for the conductor of the normalisation, and let $D \subset X$ and $C \subset Y$ be the subschemes they define. Then it follows from the exact diagram

$$
\begin{aligned}
& \begin{array}{cccccccccc}
0 & \rightarrow & \mathscr{C} & \subset & \mathscr{O}_{X} & \rightarrow & \mathscr{O}_{D} & \rightarrow & 0 \\
& \| & & \bigcap & & \bigcap & & & &
\end{array} \\
& 0 \rightarrow \pi \mathscr{C}_{Y} \subset \pi_{*} \mathscr{O}_{Y} \rightarrow \varphi_{*} \mathscr{O}_{C} \rightarrow 0
\end{aligned}
$$

that

$$
\pi \cdot \mathscr{O}_{Y} / \mathscr{O}_{X}=\varphi_{+} \mathscr{O}_{C} / \mathscr{O}_{D} \text { and } \mathscr{C}=\operatorname{Ann}_{C_{X}}\left(\varphi_{+} \mathscr{O}_{C} / \mathscr{O}_{D}\right)
$$

Note that by definition of $\mathscr{C}$ it follows that $\pi_{+} \mathscr{O}_{Y} / \mathscr{O}_{X}$ is a faithful $\mathscr{O}_{D}$-module.

All this just means that $X$ is the ringed space constructed by glueing a normal variety $Y$ along a finite morphism $\varphi: C \rightarrow D$; that is, $X$ is the topological space $Y$ modulo the equivalence defined by $\varphi$, and

$$
\mathscr{O}_{X}=\operatorname{ker}\left\{\pi_{\star} \mathscr{O}_{Y} \rightarrow \varphi_{+} \mathscr{O}_{C} \rightarrow \varphi_{\star} \mathscr{O}_{C} / \mathscr{O}_{D}\right\}
$$

In general, starting from $Y$ and $\varphi$, the resulting ringed space is not necessarily a quasiprojective scheme (for example, the union of exceptional surfaces in Hironaka's famous counterexample, or the fibre bundle of cuspidal cubic curves of [Horrocks]); it is always an algebraic space by [Artin], Theorem 6.1. In my case this is not a problem, since $\omega_{X}{ }^{-1}$ will be ample.

2.2 Proposition. $X$ satisfies $S_{2}$ at a scheme-theoretic point $P \in X$ of codimension (height) $\geq 2$ if and only if every rational section of $\mathscr{O}_{X}$ regular along every irreducible codimension 1 subscheme through $P$ is regular at $P$; or, in other words, for every scheme-theoretic point $Q \in X$ of codimension $\geq 2$ such that $P \in V(Q)$ is in the closure of $Q$, the $\mathscr{O}_{X}$-submodule $\mathscr{O}_{X} \subset \mathscr{O}_{Y}$ does not have a $Q$-primary component, that is, $Q \notin \operatorname{Ass}\left(\pi, \mathscr{O}_{Y} / \mathscr{O}_{X}\right)=\operatorname{Ass}\left(\varphi, \mathscr{O}_{C} / \mathscr{O}_{D}\right)$.

Proof. The first sentence is standard, see for example [YPG, 3.17-18]; since $Y$ is normal, a rational section of $\mathscr{O}_{X}$ regular along every irreducible codimension 1 subscheme through $P$ extends as a regular section of $\pi_{1} \mathscr{O}_{Y}$, so that the $Q$-primary part of $\pi_{*} \mathscr{O}_{Y} / \mathscr{O}_{X}$ is exactly the obstruction to $\operatorname{depth}_{Q} \mathscr{O}_{X} \geq 2$.

The proposition means the following: think of $X$ as constructed by glueing a normal $n$-dimensional variety $Y$ along a finite morphism $\varphi: C \rightarrow D$. Then for $X$ to satisfy $S_{2}$, the glueing must all be forced by what happens in codimension 1 ; that is, $C$ and $D$ have pure codimension 1 , and $\mathscr{O}_{X} \subset \pi_{k} \mathscr{G}_{Y}$ is determined by the local subrings $\mathscr{C}_{D, \Gamma} \subset\left(\varphi \mathscr{G}_{C}\right)_{\Gamma}$ at the generic point of each component $\Gamma$ of $D$, so that a given 
$f \in \pi_{*} \mathscr{O}_{Y}$ belongs to $\mathscr{O}_{X}$ if and only if its image $\bar{f} \in \varphi_{*} \mathscr{O}_{C}$ belongs to $\mathscr{O}_{D, \Gamma}$ at the generic point of each $\Gamma$.

Note that if $C$ is reduced and $\varphi: C \rightarrow D$ separable, it follows that the glueing is entirely geometric in nature, that is, $\mathscr{O}_{X}$ consists of all functions on $Y$ constant on the general geometric fibres of $\varphi$. See 4.2-3 for use of these ideas.

The $S_{2}$ condition (saturated in codimension 1, reflexive, divisorial, etc.) is discussed for example in [YPG, 3.17-18] or in [Kollár], 2.2. I make constant use of the fact that if $\mathscr{O}_{X}$ is $S_{2}$ then $\mathscr{O}_{X} \subset \pi_{*} \mathscr{O}_{Y}$ is an intersection of codimension 1 primary components, and hence so is $\mathscr{C}=\mathscr{H} a m\left(\pi_{*} \mathscr{O}_{Y}, \mathscr{O}_{X}\right)$. Thus each of $\mathscr{O}_{X} / \mathscr{C}, \pi_{*} \mathscr{O}_{Y} / \mathscr{C}$ and $\pi_{*} \mathscr{O}_{Y} / \mathscr{O}_{X}$ are $S_{1}$ or torsion-free as $\mathscr{O}_{D}$-modules. This condition can be interpreted as saying that $\mathscr{O}_{D}$ is normal under $\mathscr{O}_{C}$, that is, rational sections of $\mathscr{O}_{D}$ in $\varphi_{*} \mathscr{O}_{C}$ are already in $\mathscr{O}_{D}$. Similar remarks apply to the duals $\omega_{D}, \varphi_{*} \omega_{C}$ and ker $\operatorname{Tr}_{C / D}$ (see Remark 2.9), so that many questions reduce to the stalks of these sheaves at generic points of $D$.

The $S_{2}$ isation or saturation of a coherent sheaf $\mathscr{F}$ on a scheme $Z$ is the unique sheaf $\mathscr{F}^{\prime}$ with an $\mathscr{O}_{Z}$-linear morphism $\mathscr{F} \rightarrow \mathscr{F}^{\prime}$ such that $\mathscr{F}^{\prime}$ is $S_{2}$. This is the same thing as the reflexive hull or double dual of $\mathscr{F}$ if $Z$ is a normal variety.

2.3 Proposition ("Subadjunction", compare [Mumford]). $\omega_{Y}$ is determined by $\omega_{X}$ and the conductor as follows:

$$
\pi_{*} \omega_{Y}=\mathscr{H}_{\mathrm{C}_{C X}}\left(\pi_{*} \mathscr{O}_{Y}, \omega_{X}\right) ;
$$

(the $\pi_{*} \mathscr{O}_{Y}$-module structure is given by multiplication in the first entry of the $\mathscr{H}_{0}$ ). The right-hand side is the biggest $\pi_{*} \mathscr{O}_{Y}$-module contained in $\omega_{X}$. It coincides with the $S_{2}$ isation of the $\mathscr{O}_{Y}$-module $\mathscr{C} \cdot \omega_{X}$ if $X$ is Gorenstein in codimension 1 (in fact even without this hypothesis, see 3.6, Step 3).

In particular, if $X$ satisfies $S_{2}$ and $\omega_{X}$ is invertible then

$$
\pi_{+} \omega_{Y}=\mathscr{C} \cdot \omega_{X} \text { and } \pi^{*} \omega_{X}=\mathscr{H} \operatorname{lom}\left(\mathscr{C}, \omega_{Y}\right)=\omega_{Y}(C) \text {, }
$$

where $\omega_{Y}(C)$ is the $S_{2}$ isation of $\omega_{Y} \otimes \mathscr{O}_{Y}(C)$, that is, the divisorial sheaf on $Y$ of rational sections of $\omega_{Y}$ with poles along $C$.

2.4 Proof. The adjunction formula in the first sentence is standard use of duality (see for example Proposition 2.11 of the Appendix for the case of projective $k$-schemes). A homomorphism $\alpha: \pi_{+} \mathscr{O}_{Y} \rightarrow \omega_{X}$ is of course determined by $\alpha(1)$, so that

$$
\mathscr{H}_{o m_{n_{X}}}\left(\pi_{*} \mathscr{O}_{Y}, \omega_{X}\right)=\left\{s \in \omega_{X} \mid f s \in \omega_{X} \text { for all } f \in \pi_{*} \mathscr{O}_{Y}\right\} \text {, }
$$

which is equal to $\mathscr{C} \cdot \omega_{X}$ on the locus where $\omega_{X}$ is locally free. The rest is easy: $\mathscr{C}$ satisfies $S_{2}$ by the above, and $\omega_{Y}$ by [C3-f, App. to $\S 1$, Theorem 7]; and two coherent sheaves satisfying $S_{2}$ that coincide in codimension 1 are equal.

Q.E.D.

2.5 Etymology. The name subadjunction is explained as follows: for an irreducible plane curve $X$ of degree $d$, the canonical class of the resolution or normalisation $Y$ is the sheaf of $\mathscr{O}_{Y}$-modules generated by $\mathscr{C} \cdot \mathscr{O}(d-3)$, and the conductor ideal 
$\mathscr{C}$ itself is determined in terms of adjoint forms, that is, forms vanishing to order $m-1$ at every $m$-fold point of $X$, including infinitely near points (this goes back to Brill and Noether around 1870, and is also the subject in the 1950s of Gorenstein's thesis and sections in Kodaira's papers on surfaces). In higher dimension, the canonical class and plurigenera of the resolution of an irreducible hypersurface with arbitrary singularities correspond to adjunction ideals that can't be described in such simple terms; however, by the proposition, the canonical class of the normalisation is determined by the conductor ideal, given exactly as in the curve case by conditions in codimension 1 , the subadjunction conditions.

Subadjunction played a foundational role in the dark ages before Kähler differentials and the Grothendieck dualising sheaf: the canonical class of a nonsingular projective variety $V$ was often defined in terms of subadjunction applied to a generic projection of $V$ as hypersurface. It's clear from Enriques' discussion of subadjunction and adjunction for singular surfaces in 3-spaces in [Enriques, Ch. III, §§6-7] that he understood pretty well the case of ordinary multiple points, and the difficulties of working with worse isolated singularities; but it's curious that he does not seem to know Du Val's work, the most substantial result known at the time.

2.6 The next result solves the converse problem of determining $\omega_{X}$ in terms of $Y$ and $\varphi: C \rightarrow D$.

Theorem. Assume that $X$ is $S_{2}$, so that in particular $C$ and $D$ have pure dimension $n-1$. Then applying the cohomological $\partial$-functor $\mathscr{E}_{x t_{i x}^{+}}^{+}\left(-, \omega_{X}\right)$ to the exact diagram $(*)$ at the start of $\$ 2$ gives the commutative diagram with exact rows

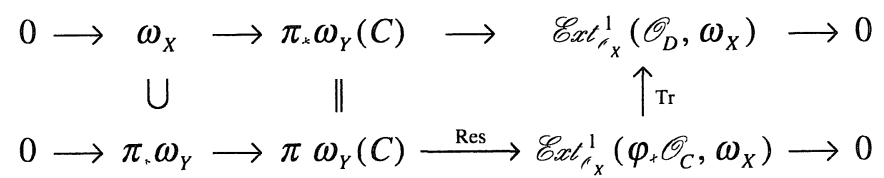

Moreover,

$$
\begin{gathered}
\omega_{D}=S_{2} \text { isation of } \mathscr{E}_{x t_{\mu_{X}}^{1}}\left(\mathscr{O}_{D}, \omega_{X}\right) \\
\varphi_{r} \omega_{C}=S_{2} \text { isation of } \mathscr{E}_{x t_{\digamma_{X}}^{1}}\left(\varphi_{k} \mathscr{O}_{C}, \omega_{X}\right) ;
\end{gathered}
$$

the $S_{2}$ isation does nothing if $X$ is Cohen-Macaulay, so that

$$
\omega_{D}=\mathscr{E}_{x t_{x}^{1}}^{1}\left(\mathscr{O}_{D}, \omega_{X}\right) \text { and } \varphi_{+} \omega_{C}=\mathscr{E}_{x t_{\pi_{X}}^{1}}\left(\varphi_{+} \mathscr{O}_{C}, \omega_{X}\right) \text {. }
$$

In more detail, this means the following:

(1) The composite of the surjection in the exact sequence

$$
0 \rightarrow \pi_{1} \omega_{Y} \rightarrow \pi, \omega_{Y}(C) \rightarrow \mathscr{E}_{x c_{c}^{1}}^{1}\left(\varphi, \mathscr{O}_{C}, \omega_{X}\right) \rightarrow 0
$$

and the $S_{2}$ isation $\mathscr{E}_{x l_{1}^{\prime}}^{1}\left(\varphi_{*} \mathscr{O}_{C}, \omega_{X}\right) \subset \varphi \cdot \omega_{C}$ is a canonically defined "Poincaré residue" map $\operatorname{Res}=\operatorname{Res}_{Y, C}: \omega_{Y}(C) \rightarrow \omega_{C}$. 
(2) The trace map $\operatorname{Tr}=\operatorname{Tr}_{C / D}: \varphi_{r} \omega_{C} \rightarrow \omega_{D}$, which is canonically defined and surjective, fits into a diagram

$$
\begin{array}{ccc}
\mathscr{E}_{x t_{\tilde{F}_{X}}^{1}}^{1}\left(\mathscr{O}_{D}, \omega_{X}\right) & \subset & \omega_{D} \\
\uparrow_{\mathrm{Tr}} & & \uparrow_{\mathrm{Tr}} \\
\mathscr{E}_{x t_{C_{X}}^{1}}\left(\varphi_{*} \mathscr{O}_{C}, \omega_{X}\right) & \subset & \varphi_{*} \omega_{C} .
\end{array}
$$

(3) In these terms,

$$
\omega_{X}=\operatorname{ker}\left\{\pi_{*} \omega_{Y}(C) \stackrel{\operatorname{Res}}{\longrightarrow} \varphi_{*} \omega_{C} \stackrel{\operatorname{Tr}}{\longrightarrow} \omega_{D}\right\} .
$$

2.7 Proof. This all follows formally from the adjunction properties of predualising sheaves (discussed in the appendix). First of all, clearly $\mathscr{H}_{0}\left(\mathscr{O}_{D}, \omega_{X}\right)=$ $\mathscr{H a m}\left(\mathscr{O}_{C}, \omega_{X}\right)=0$ since $\omega_{X}$ is torsion-free; $\mathscr{H}_{a_{C_{X}}}\left(\mathscr{O}_{X}, \omega_{X}\right)=\omega_{X}$ is obvious, and subadjunction gives $\mathscr{H}_{\text {om } \mathrm{f}_{X}}\left(\pi_{*} \mathscr{O}_{Y}, \omega_{X}\right)=\pi_{*} \omega_{Y}$. Next,

$$
\operatorname{Hom}_{C_{X}}\left(\mathscr{C}, \omega_{X}\right)=\mathscr{H}_{a m_{f_{X}}}\left(\pi_{*} \mathscr{C}_{Y}, \omega_{X}\right)=\pi_{*} \omega_{Y}(C) \text {. }
$$

This holds because $\mathscr{C}=\pi_{*} \mathscr{C}_{Y}$ is a $\pi_{*} \mathscr{O}_{Y}$-module, so any $\mathscr{O}_{X}$-linear homomorphism from it can only map into a $\pi_{*} \mathscr{O}_{Y}$-submodule of $\omega_{X}$, that is, into $\pi_{*} \omega_{Y}$; thus the middle term is

$$
\operatorname{Ham}_{\pi_{*} \varsigma_{Y}}\left(\pi_{*} \mathscr{C}_{Y}, \pi_{*} \omega_{Y}\right)=\pi_{*} \omega_{Y}(C) .
$$

Next, $\mathscr{E}_{x t t_{X}^{1}}\left(\mathscr{G}_{X}, \omega_{X}\right)=0$ follows from basic properties of $\mathscr{E}_{x t} s$, since the stalks of $\mathscr{O}_{X}$ are projective. As just explained, the two functors $\mathscr{H}_{0 m_{n_{X}}}\left(-, \omega_{X}\right)$ and $\mathscr{H}_{a_{\pi_{+} \epsilon_{Y}}}\left(-, \pi_{+} \omega_{Y}\right)$ coincide on $\pi_{*} \mathscr{Q}_{Y}$-modules, so that the fact that the stalks of $\pi_{+} \mathscr{O}_{Y}$ are projective over $\pi_{k} \mathscr{O}_{Y}$ implies that also $\mathscr{E}_{x t_{\pi x}^{1}}^{1}\left(\pi_{+} \mathscr{O}_{Y}, \omega_{X}\right)=0$.

Finally, the adjunction formula Theorem 2.12 of the Appendix gives that $\omega_{D}$ is the $S_{2}$ isation of $\mathscr{E}_{x l_{\pi_{x}}^{1}}\left(\mathscr{O}_{D}, \omega_{X}\right)$ and $\varphi_{*} \omega_{C}$ that of $\mathscr{E}_{x t_{k}^{1}}^{1}\left(\varphi, \mathscr{O}_{C}, \omega_{X}\right)$.

Therefore, applying $\mathscr{E}_{x l_{c}^{*}}\left(-, \omega_{X}\right)$ to $\left(^{*}\right)$ gives the diagram $\left(^{* *}\right)$ of the theorem.

Q.E.D.

2.8 Corollary. Equivalent conditions:

(i) The predualising sheaf $\omega_{X}$ is an invertible $\mathscr{O}_{X}$-module.

(ii) $\omega_{X}$ is invertible in codimension 1 , and for all $P \in Y$ there exists an element $s \in \operatorname{ker}\left(\operatorname{Tr}_{C / D} \circ \operatorname{Res}_{Y, C}\right): \pi_{*} \omega_{Y}(C) \rightarrow \omega_{D}$ such that $\omega_{Y}(C)=\mathscr{G}_{Y} \cdot$ s near $P$.

(iii) $\omega_{X}$ is invertible in codimension $1, \omega_{Y}(C)$ is invertible, and for all $P \in C$ there exists a basis element $s \in \operatorname{ker} \operatorname{Tr}_{C I D}: \mathscr{E}_{x l_{1}^{1}}^{1}\left(\varphi_{*} \mathscr{O}_{C}, \omega_{X}\right) \rightarrow \mathscr{E}_{x l_{1}}^{1}\left(\mathscr{O}_{D}, \omega_{X}\right)$ such that

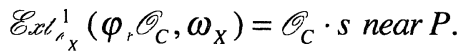

(iv) $\omega_{Y}(C)$ is an invertible $\mathscr{O}_{Y}$-module and

$$
\operatorname{ker}\left\{\operatorname{Tr}_{C / D}: \mathscr{E}_{x l_{1}^{\prime}}^{1}\left(\varphi_{r} \mathscr{O}_{C}, \omega_{X}\right) \rightarrow \mathscr{E}_{x l_{1}^{\prime}}^{1}\left(\mathscr{O}_{D}, \omega_{X}\right)\right\}
$$


is an invertible $\mathscr{O}_{D}$-module.

Note that when $X$ is Cohen-Macaulay, conditions (iii) and (iv) refer simply to the kernel of $\operatorname{Tr}_{C / D}: \varphi_{*} \omega_{C} \rightarrow \omega_{D}$.

Proof. (i) $\Rightarrow$ (ii) or (iv) is clear, since $\omega_{X}=\mathscr{O}_{X} \cdot s$ implies that $\omega_{Y}(C)=$ $\pi^{*} \omega_{X}=\mathscr{O}_{Y} \cdot s$ by Proposition 2.3, and $s \in \operatorname{ker}(\operatorname{Tr} \circ \operatorname{Res})$ by Theorem 2.6; and $s$ maps to an $\mathscr{O}_{D}$-basis of ker $\operatorname{Tr}_{C / D}$. (ii) $\Leftrightarrow$ (iii) is clear by the surjectivity of Res in (**), since if $s \in \omega_{Y}(C)$ maps to $\bar{s}=\operatorname{Res} s \in \mathscr{E}_{x t_{\mathscr{O}_{X}}^{1}}\left(\varphi_{*} \mathscr{O}_{C}, \omega_{X}\right)$ then $s$ is an $\mathscr{O}_{Y}$-basis of $\omega_{Y}(C)$ if and only if $\bar{s}$ is an $\mathscr{O}_{C}$-basis of $\mathscr{E}_{x t_{\epsilon_{X}}^{1}}\left(\varphi_{*} \mathscr{O}_{C}, \omega_{X}\right)$.

(ii) $\Rightarrow$ (i) Let $Q \in X$. First suppose that $s \in \operatorname{ker}\left(\operatorname{Tr}_{C / D} \circ \operatorname{Res}_{Y, C}\right)$ is an $\mathscr{O}_{Y}$-basis of $\omega_{Y}(C)$ near $P$ for every $P$ lying over $Q$; required to prove that $\mathscr{O}_{X} \cdot s=\omega_{X}$ near $Q$. This is true after localising at the generic point of a codimension 1 subvariety $\Gamma \subset X$ : for by assumption, $\omega_{X, \Gamma}$ is locally free, and if $s \in m_{\Gamma} \cdot \omega_{X, \Gamma}$ then $s$ could not be a basis of $\omega_{Y}(C)$ above $\Gamma$. The result then follows from the fact that $\omega_{X}$ is $S_{2}$.

By (ii), for each $P \mapsto Q$ there is an element

$$
s_{P} \in \operatorname{ker}\left(\operatorname{Tr}_{C / D} \circ \operatorname{Res}_{Y, C}\right): \pi_{*} \omega_{Y}(C) \rightarrow \omega_{X}
$$

that is an $\mathscr{O}_{Y}$-basis of $\omega_{Y}(C)$ near $P$. If the residue field of $\mathscr{O}_{X, Q}$ is infinite, or big enough compared with the number of $P$, then a suitable linear combination of the $s_{P}$ with coefficients in $\mathscr{O}_{X, Q}$ will be a basis at every $P$.

It's not hard to deal with the case that $\mathscr{O}_{X, Q}$ has a finite residue field by making a finite faithfully flat extension $\mathscr{O}_{X . Q} \subset \mathscr{O}_{X^{\prime}, Q}$ to increase the residue field, concluding that $\omega_{X^{\prime}}$ is locally free by the above argument, and then using the fact that a finite module over a local ring which becomes free after a faithfully flat extension was already free (because free = flat, e.g., [Bourbaki, Cor. II.3.2.5.2]).

(iv) $\Rightarrow$ (i) Outside $D$ and $C$, obviously $\omega_{X}=\omega_{Y}=\omega_{Y}(C)$; near $D$, the exact diagram $(* *)$ can be rewritten

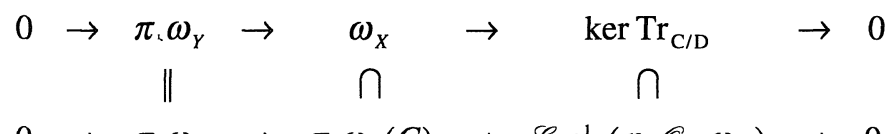

$$
\begin{aligned}
& 0 \rightarrow \pi, \omega_{Y} \rightarrow \pi_{*} \omega_{Y}(C) \rightarrow \mathscr{E}_{x t_{x}^{1}}^{1}\left(\varphi_{*} \mathscr{O}_{C}, \omega_{X}\right) \rightarrow 0 .
\end{aligned}
$$

The kernel in the top left is $\omega_{X} \cap \pi \cdot \omega_{Y}=\pi \omega_{Y}$.

Pick an $\mathscr{O}_{D}$-basis $\bar{s} \in \operatorname{ker} \operatorname{Tr}_{C / D}$, and $\bar{s} \in \omega_{X}$ mapping to it. I claim that $\mathscr{O}_{X} \cdot s=\omega_{X}$. Since both $\mathscr{O}_{X} \cdot s$ and $\omega_{X}$ are $S_{2}$, it's enough to prove this in codimension 1 . Now $\mathscr{C} \cdot \omega_{X} \subset \pi \cdot \omega_{Y}$ by Proposition 2.3, and I will prove in 3.6, Step 3 that equality holds in codimension 1 . Now on the locus where $\pi \cdot \omega_{Y}=\mathscr{C} \cdot \omega_{X}$, I have $\operatorname{ker} \operatorname{Tr}_{C / D}$ $=\omega_{X} /\left(\mathscr{C} \cdot \omega_{X}\right)=\omega_{X} \otimes \mathscr{O}_{D}$, so that

$$
\bar{s} \text { bases ker } \operatorname{Tr}_{C / D} \Rightarrow s \text { bases } \omega_{X}
$$

follows from Nakayama's lemma.

Q.E.D. 
2.9 Remark. Writing down the trace map $\operatorname{Tr}_{C I D}$ is an activity that takes place at the generic point of each component of $D$, and reduces there to duality for Artinian local rings. Each of $\varphi_{+} \omega_{C}$ and $\omega_{D}$ is torsion-free, so a subsheaf of its rational sections (the sheaf made up of direct sums of generic stalks). If $\Gamma$ is a component of $D$, then the generic stalk $\mathscr{O}_{D, \Gamma}$ is an Artinian local ring, with dualising module $\omega_{D, \Gamma}$, and $\operatorname{Tr}: \varphi_{+} \omega_{C, \Gamma} \rightarrow \omega_{D, \Gamma}$ is just the $\mathscr{O}_{D, \Gamma}$-dual of the inclusion $\mathscr{O}_{D, \Gamma} \subset \varphi_{*} \mathscr{O}_{C, \Gamma}$. Thus ker $\operatorname{Tr}_{C / D}$ can be described as the subsheaf

$$
\text { ker } \operatorname{Tr}_{C / D}=\left\{s \in \varphi_{*} \omega_{C} \mid \operatorname{Tr}\left(s_{\Gamma}\right)=0 \in \omega_{D, \Gamma} \text { for all } \Gamma\right\} \subset \varphi_{*} \omega_{C} .
$$

Although $\operatorname{Tr}_{C / D}$ has this "birational" description, the condition for $\operatorname{ker} \operatorname{Tr}_{C / D}$ to be invertible as an $\mathscr{O}_{D}$-module is nevertheless a delicate biregular question, and understanding this in a special case is the main point of $\S 4$. A subtle point that causes a lot of confusion is that $\operatorname{Tr}_{C / D}$ is only linear over $\mathscr{O}_{D}$; however, the subring $\mathscr{O}_{D}$ is the unknown in my calculations in $\$ 4$.

\section{Appendix to \$2. Adjunction for a Finite Morphism}

2.10 This section is a technical digression. $\pi: Y \rightarrow X$ is a finite morphism of schemes, not necessarily surjective. By [Hartshorne, Ch. II, Ex. 5.17, (e)], $\pi_{*}$ identifies the category of $\mathscr{O}_{Y}$-modules on $Y$ with that of $\mathscr{O}_{X}$-modules on $X$ together with an action of $\pi_{*} \mathscr{O}_{Y}$; the map back is the module-to-sheaf construction $M \mapsto \tilde{M}$ generalised from affine schemes to affine morphisms. I just say $\mathscr{O}_{Y}$-module from now on.

The right adjoint of $\pi_{*}$ takes an $\mathscr{O}_{X}$-module $\mathscr{G}$ into $\mathscr{H}_{m_{m_{X}}}\left(\pi_{*} \mathscr{O}_{Y}, \mathscr{G}\right)$, made into an $\mathscr{O}_{Y}$-module by multiplication in the source by elements of $\pi_{*} \mathscr{O}_{Y}$ : as written in Holy Scriptures [Grothendieck-Hakim, Grothendieck-Hartshorne, §4], if $\mathscr{C}$ is the category of $A$-modules, and $T: \mathscr{C} \rightarrow \underline{\mathrm{Ab}}$ a contravariant functor, then $T(M)$ has a canonical $A$-module structure with $a \in A$ acting by $T\left(\mu_{a}\right)$, where $\mu_{a}$ is the homothety of multiplication by $a$; this must be borne in mind throughout. For an $\mathscr{O}_{Y}$-module $\mathscr{F}$ and an $\mathscr{O}_{X}$-module $\mathscr{G}$, there is a canonical bifunctorial isomorphism

$$
\operatorname{Hom}_{c_{x}}\left(\pi_{*} \mathscr{F}, \mathscr{G}\right)=\operatorname{Hom}_{c_{y}}\left(\mathscr{F}, \mathscr{H}_{m_{c_{x}}}\left(\pi_{*} \mathscr{O}_{Y}, \mathscr{G}\right)\right) \text {. }
$$

To use easy characterisations of the predualising sheaf $\omega_{X}$ I work here only with quasiprojective $k$-schemes. A more sophisticated definition of the dualising complex makes everything work more generally, somewhat tautologically; but the existence of the dualising complex is hard in general.

Recall from [Hartshorne, Ch. III, §7] that the predualising sheaf $\omega_{X}$ on an $n$-dimensional projective scheme $X$ is determined by the following universal mapping property: there is a $k$-linear map "trace" $t: H^{n}\left(X, \omega_{X}\right) \rightarrow k$, and for every coherent sheaf $\mathscr{F}$ on $X$, any $k$-linear map $H^{n}(X, \mathscr{F}) \rightarrow k$ is induced by a morphism $\mathscr{F} \rightarrow \omega_{X}$, so that $H^{n}(X, \mathscr{F})$ d $\operatorname{Hom}\left(\mathscr{F}, \omega_{X}\right)$. 
2.11 Proposition ([Hartshorne, Ch. III, Ex. 7.2]). Let $\pi: Y \rightarrow X$ be a finite morphism of projective $k$-schemes with $\operatorname{dim} X=\operatorname{dim} Y$. Then

$$
\omega_{Y}=\operatorname{Hom}_{C_{X}}\left(\pi_{*} \mathscr{O}_{Y}, \omega_{X}\right)
$$

and the map $\omega_{Y} \rightarrow \omega_{X}$ defined by $\alpha \mapsto \alpha(1)$ is the trace map for $\pi$.

Proof. Let $\mathscr{F}$ be any coherent sheaf on $Y$; then (omitting the $\pi_{*}$ for clarity), I get

$$
\begin{aligned}
H^{n}(Y, \mathscr{F})=H^{n}(X, \mathscr{F}) \stackrel{\mathrm{d}}{-} \operatorname{Hom}_{\mathscr{C}_{X}}\left(\mathscr{F}, \omega_{X}\right) \\
=\operatorname{Hom}_{\mathscr{C}_{Y}}\left(\mathscr{F}, \mathscr{H}_{a_{\mathscr{C}_{\mathrm{X}}}}\left(\mathscr{C}_{Y}, \omega_{X}\right)\right) .
\end{aligned}
$$

Therefore $\omega_{Y}=\mathscr{H}_{a_{C_{\mathrm{X}}}}\left(\mathscr{\mathscr { O }}_{Y}, \omega_{X}\right)$ satisfies the universal property of a dualising sheaf on $Y$.

Q.E.D.

2.12 Theorem (Adjunction formula). Let $X$ be a purely $n$-dimensional quasiprojective scheme, $\omega_{X}$ a predualising sheaf for $X$ [Hartshorne, Ch. III, §7] and $\pi: Y \rightarrow X$ a finite morphism, with $Y$ purely of dimension $n-r$.

(1) Suppose that $X$ is Cohen-Macaulay. Then the predualising sheaf of $Y$ is given by

$$
\omega_{Y}=\mathscr{E}_{x t_{t_{X}}^{r}}\left(\pi_{*} \mathscr{O}_{Y}, \omega_{X}\right)
$$

(2) Suppose that $X$ is Cohen-Macaulay at every codimension 1 point of $Y$ (this holds in particular if $X$ satisfies Serre's condition $\left.S_{r+1}\right)$. Then $\omega_{Y}$ is the $S_{2}$ isation (see 2.2) of $\mathscr{E}_{x t_{K_{X}}^{r}}\left(\pi_{\star} \mathscr{G}_{Y}, \omega_{X}\right)$.

Proof. (1) Suppose $X \subset \mathbb{P}^{N}=\mathbb{P}$ has codimension $s$. Then

$$
\omega_{Y}=\mathscr{E}_{x t_{\mathrm{p}}^{r+s}}^{r+s}\left(\mathscr{O}_{Y}, \omega_{\mathrm{P}}\right) \text {. }
$$

This $\mathscr{E}_{x t}$ is the $(r+s)$ th homology sheaf of the complex $\mathscr{H}_{a m_{\pi_{\mathrm{p}}}}\left(\mathscr{O}_{Y}, \mathscr{I}^{\cdot}\right)$, where $\omega_{\mathrm{P}} \rightarrow \mathscr{F} \cdot$ is an injective resolution. But $\mathscr{O}_{Y}$ is a sheaf of $\mathscr{O}_{X}$-modules, so that by the above, I can $\mathscr{L}_{\text {om }}$ via $\mathscr{O}_{X}$ :

$$
\operatorname{Hom}_{\rho_{\mathbf{p}}}\left(\mathscr{G}_{Y}, \mathscr{I} \cdot\right)=\operatorname{Hom}_{c_{X}}\left(\mathscr{O}_{Y}, \mathscr{H}_{m_{f_{\mathbf{P}}}}\left(\mathscr{O}_{X}, \mathscr{I} \cdot\right)\right) .
$$

I claim that the inner complex $\mathscr{J}^{\cdot}=\mathscr{H}_{m_{\mu_{0}}}\left(\mathscr{O}_{X}, \mathscr{I}^{\prime}\right)$ is essentially an injective resolution of $\omega_{X}$ shifted by $s$. Indeed, it is easy to see that $\mathscr{I}^{l}$ injective over $\mathscr{O}_{\mathbf{P}}$ implies that $\mathscr{J}^{\imath}=\mathscr{H}_{\mathrm{O} \mathrm{m}_{\mathrm{p}}}\left(\mathscr{G}_{X}, \mathscr{I}^{\imath}\right)$ is injective as $\mathscr{O}_{X}$-module. Also, because $X$ is Cohen-Macaulay, the complex $\mathscr{J}^{\cdot}$ has cohomology

$$
\mathscr{E}_{x t_{\mathrm{p}}^{l}}\left(\mathscr{O}_{X}, \omega_{\mathrm{p}}\right)= \begin{cases}\omega_{X} & \text { if } i=s, \\ 0 & \text { if } i \neq s .\end{cases}
$$

Thus $\mathscr{J}^{\prime}$ can be written as a direct sum of two complexes $\mathscr{J}_{1} \oplus \mathscr{J}_{2}$, where $\mathscr{J}_{1}$ is an exact complex of injective $\mathscr{O}_{x}$-modules in degree $[0, s]$ and $\mathscr{J}_{2}$ an injective resolution of $\omega_{X}$ starting in degree $s$. Thus 


$$
\mathscr{E}_{x t_{C_{\mathbf{P}}}^{r+s}}\left(\mathscr{C}_{Y}, \omega_{\mathbf{P}}\right)=\mathscr{E}_{x t_{C_{X}}^{r}}\left(\mathscr{C}_{Y}, \omega_{X}\right) .
$$

(2) follows at once from the fact that $\omega_{Y}$ is $S_{2}$, and by what I've just said, coincides with $\mathscr{E}_{x t t_{\mathscr{C}_{X}}^{r}}\left(\mathscr{\mathscr { O }}_{Y}, \omega_{X}\right)$ in codimension 1 .

Q.E.D.

2.13 Examples. Here is the well-known case of the theorem with $r=1$, and a counterexample pointed out by Kollár to show that the $S_{2}$ isation is really needed in Theorem 2.12.

(a) Suppose $Y \subset X$ is a Cartier divisor; then applying $\mathscr{E}_{x t} s$ to the exact sequence $0 \rightarrow \mathscr{O}_{X}(-Y) \rightarrow \mathscr{O}_{X} \rightarrow \mathscr{O}_{Y} \rightarrow 0$, gives

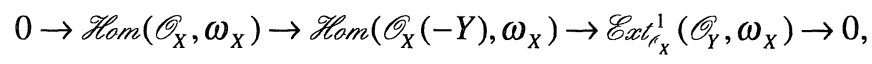

that is, $\mathscr{E}_{x t_{\Lambda_{X}}^{1}}\left(\mathscr{O}_{Y}, \omega_{X}\right)=\omega_{X}(Y) / \omega_{X}=\omega_{X}(Y) \otimes \mathscr{O}_{Y}$. Hence if $X$ is $S_{2}$ then

$$
\omega_{Y}=S_{2} \text { isation of } \mathscr{E}_{x t_{C_{X}}}^{1}\left(\mathscr{O}_{Y}, \omega_{X}\right)=S_{2} \text { isation of } \omega_{X}(Y) \otimes \mathscr{O}_{Y} \text {. }
$$

(b) Let $X$ be the projective cone over a normally embedded Abelian surface $A$ and $Y$ a hypersection through the vertex point $O$. Then $\omega_{X} \cong \mathscr{O}_{X}(-Y)$ and $\mathscr{E}_{x t_{1}}^{1}\left(\mathscr{O}_{Y}, \omega_{X}\right) \cong \mathscr{O}_{Y}$ by the argument of (a) above. But $Y$ is not normal, so that $\mathscr{O}_{Y}$ is not $S_{2}$; here $\omega_{Y}=\mathscr{O}_{\tilde{Y}}$ where $\pi: \tilde{Y} \rightarrow Y$ is the normalisation.

(c) Let $Y=Y_{1}+Y_{2}$ be Cartier divisors on $X$ as in (a). Then the theorem applies to the inclusion morphism $Y_{1} \subset Y$, giving

$$
\omega_{Y_{1}}=\mathscr{H} m_{r_{Y}}\left(\mathscr{O}_{Y_{1}}, \omega_{Y}\right) \text {. }
$$

It's not hard to evaluate this to be $=\omega_{Y}\left(-Y_{2}\right) \otimes \mathscr{C}_{Y_{1}}$.

\section{§3. What Happens in Codimension 1}

Duality for finite modules over a local Artinian (0-dimensional) ring is almost as easy and explicit as for vector spaces over a field. The aim of this section is to apply this duality to complete the proof of Corollary 2.8 , (ii) or (iv) $\Rightarrow$ (i), to translate the condition for $X$ to be Gorenstein in codimension 1 into a more explicit form, and to solve this condition in the simplest cases.

Most of the material is copied more or less directly from [Serre, Ch. IV, §11], although my category is closer to the natural level of generality sought by the true Bourbakist.

3.1 Notation and assumptions. Except where stated otherwise, I localise everything throughout $\S 3$ at a codimension 1 point of $X$, that is, at the generic point of an irreducible codimension 1 locus $\Gamma \subset X$. Suppose that $C \subset Y$ localised above $\Gamma \subset X$ has the decomposition into prime divisors $C=\sum n_{E} E$. Thus $\mathscr{O}_{X}$ is a reduced 1-dimensional local ring, and is a subring of $\mathscr{C}_{Y}$, which is a product of DVRs: 


$$
\mathscr{O}_{Y}=\prod R_{E} \text {, where } R_{E}=\mathscr{O}_{Y, E} \text { is a DVR with local parameter } t_{E} .
$$

I suppress $\pi_{*}$ throughout $\S 3$. Write $m=m_{\Gamma} \subset \mathscr{O}_{X}$ for the maximal ideal and $K=k(\Gamma)=\mathscr{O}_{X} / m$ for its residue field, the function field of the subscheme $\Gamma$; note that the residue field $k(E)=R_{E} /\left(t_{E}\right)$ of each localisation $R_{E}$ of $\mathscr{O}_{Y}$ is a finite extension of $K$. The geometric picture of the normalisation is "unzipping" $X$ along its codimension 1 singular locus, and in general the set-theoretic multiple locus of $Y \rightarrow X$ can be a ramified (or even inseparable) cover $\amalg E \rightarrow \Gamma$.

The conductor $\mathscr{C}$ has finite colength in $\mathscr{O}_{X}$ and $\mathscr{O}_{Y}$, and the quotients $\mathscr{O}_{D}=\mathscr{O}_{X} / \mathscr{C}$ and $\mathscr{O}_{C}=\mathscr{O}_{Y} / \mathscr{C}$ are Artinian rings, with $\mathscr{O}_{D}$ local and $\mathscr{O}_{C}=\prod A_{E}$ a product of rings of the form

$$
A_{E}=\mathscr{O}_{n E}=R_{E} /\left(t_{E}^{n_{E}}\right) \text { with } n_{E} \geq 1 .
$$

Since $\mathscr{O}_{D}$ is Artinian and local, the stalk $\omega_{D}$ of the dualising sheaf at the generic point of $\Gamma$ is the dualising module of $\mathscr{O}_{D}$, that is, $\omega_{D}=\operatorname{Hom}_{k_{0}}\left(\mathscr{O}_{D}, k_{0}\right)$ if $\mathscr{O}_{D}$ contains a field $k_{0}$ such that $k_{0} \subset K$ if a finite extension (hands up those who don't remember how the $\mathscr{O}_{D}$-module structure in the Hom is defined!); and, quite generally, $\omega_{D}$ is the injective hull of the residue field $K$ as $\mathscr{O}_{D}$-module. Thus $\operatorname{Hom}_{k_{0}}\left(-, k_{0}\right)$ in the $k_{0}$-algebra case, or Hom ${ }_{C_{D}}\left(-, \omega_{D}\right)$ quite generally, is the dualising functor for $\mathscr{O}_{D}$-modules, and I write $M \stackrel{\text { d }}{-} N$ to mean $M=\operatorname{Hom}_{\mathscr{C}_{D}}\left(N, \omega_{D}\right)$

In this section the length $\ell(M)$ of a module $M$ always means its length as an $\mathscr{O}_{D}$-module, so that, for example, the residue field $k\left(R_{E}\right)$ has length $\ell\left(k\left(R_{E}\right)\right)=\left[k\left(R_{E}\right): K\right]$, and $A_{E}$ in the preceding display has length $\ell\left(A_{E}\right)=n_{E} \cdot\left[k\left(R_{E}\right): K\right]$; as an $A_{E}$-module, this has of course a Jordan-Hölder composition series of length only $n_{E}$ defined by the powers of $t_{E}$, with successive quotients $k\left(R_{E}\right)$.

3.2 Main theorem. In the notation and assumptions of $\S 2$, consider the two diagrams

$$
\begin{array}{rllllllllll}
\mathscr{O}_{Y}(-C)=\mathscr{C} & \subset & \mathscr{O}_{X} & \subset & \mathscr{O}_{Y} & & 0 & \subset & \mathscr{O}_{D} & \subset & \mathscr{O}_{C} \\
\omega_{Y}(C) & \supset & \omega_{X} & \supset & \omega_{Y} & & \omega_{C} & \supset & \operatorname{ker} \operatorname{Tr}_{C / D} & \supset & 0,
\end{array}
$$

the first of which consists of sheaves of $\mathscr{G}_{X}$-modules, and the second of $\mathscr{O}_{D}$-modules, the corresponding quotients by $\mathscr{C}$ or $\omega_{Y}$.

(I) Localised in codimension 1, the aligned $\subset s$ and $\supset s$ correspond to $\mathscr{O}_{D}$-dual modules

$$
\begin{array}{rll}
\mathscr{O}_{X} / \mathscr{C}=\mathscr{O}_{D} & \stackrel{d}{-} & \omega_{Y}(C) / \omega_{X}=\omega_{D}, \\
\mathscr{O}_{Y} / \mathscr{O}_{X}=\mathscr{O}_{C} / \mathscr{O}_{D} \quad \stackrel{d}{-} & \omega_{X} / \omega_{Y}=\operatorname{ker} \operatorname{Tr}_{C / D} .
\end{array}
$$


(II) The length of the $\mathscr{O}_{D}$-modules in the top line is $\leq$ that in the bottom line, $\ell\left(\mathscr{O}_{D}\right) \leq \ell\left(\mathscr{O}_{C} / \mathscr{O}_{D}\right)=\ell\left(\omega_{X} / \omega_{Y}\right)$, and

$$
\text { equality } \Leftrightarrow \mathscr{O}_{D} \cong \operatorname{ker} \operatorname{Tr}_{C / D}=\omega_{X} / \omega_{Y} \Leftrightarrow \mathscr{O}_{X} \cong \omega_{X} \text {, }
$$

that is, if and only if $\omega_{X}$ is Gorenstein in codimension 1.

In general (not localising at a codimension 1 point), the first diagram can be taken to mean duality of reflexive sheaves.

3.3 Remark. In (I), all the equalities are already known from $\S 2$, with those on the left by definition, and those on the right coming from Theorem 2.6, so that the point is to prove the duality. (II) is the famous inequality $n \leq 2 \delta$ of [Serre, Ch. IV, $\S 11]$, where

$$
n=\ell\left(\mathscr{O}_{Y}(C) / \mathscr{O}_{Y}\right)=\ell\left(\mathscr{O}_{C} / \mathscr{O}_{D}\right)+\ell\left(\mathscr{O}_{D}\right)=\sum n_{E} \cdot[k(E): K]
$$

(here $C=\sum n_{E} E$, with the $E$ prime divisors of $Y$ ), and $\delta=\ell\left(\omega_{X} / \omega_{Y}\right)$; if $\pi: Y \rightarrow X$ is the normalisation (resolution) of a singularity $P \in X$ of an irreducible projective curve over an algebraically closed field, then $\delta$ is the genus of the singularity $P \in X$, that is,

$$
\delta=\delta p_{a}=h^{0}\left(\omega_{X}\right)-h^{0}\left(\omega_{Y}\right) .
$$

3.4 The proof of the theorem follows closely the arguments of [Serre, Ch. IV, $\S 11]$, which are mainly in terms of $\pi: Y \rightarrow X$, but it's convenient to waste a page of journal space discussing the following definition, which abstracts away from the 1-dimensional schemes $X$ and $Y$, and focuses on the Artinian conductor subschemes $D$ and $C$ where the magic duality works.

Definition (half-filling of $\mathscr{O}_{C}$ ). Suppose given a field $K$, and a number of DVRs $R_{E}$, with local parameters $t_{E}$, whose residue fields $k(E)=R_{E} /\left(t_{E}\right)$ are all finite extensions of $K$; let $\mathscr{C}_{C}=\prod A_{E}$ be a product of rings of the form

$$
A_{E}=R_{E} /\left(t_{E}^{n_{E}}\right) \text { with } n_{E} \geq 1 \text {. }
$$

A part-filling of $\mathscr{O}_{C}$ is a subring $\mathscr{O}_{D} \subset \mathscr{O}_{C}$ satisfying

(i) $\mathscr{O}_{D}$ is a local ring with residue field $\mathscr{O}_{D} / m_{D}=K$;

(ii) $\mathscr{O}_{C} / \mathscr{O}_{D}$ is a faithful $\mathscr{O}_{D}$-module, that is, Ann,$_{D}\left(\mathscr{O}_{C} / \mathscr{C}_{D}\right)=0$. If in addition

(iii) $\mathscr{C}_{C} / \mathscr{O}_{D} \cong \omega_{D}$ as $\mathscr{O}_{D}$-module, then $\mathscr{O}_{D}$ is a half-filling of $\mathscr{C}_{C}$.

3.5 Remarks. (a) In practical calculations, the trickiest thing to work with is the condition that $\mathscr{C}_{D}$ is a subring, since this is nonlinear; see Proposition 3.9 and 4.4 for an example. 
(b) The local condition (i) means that the image of $\mathscr{O}_{D}$ under the composite map $\mathscr{O}_{C} \rightarrow \prod R_{E} \rightarrow \prod K_{E}$ is the diagonal $K \subset \prod K_{E}$. This is equivalent to saying that $\operatorname{dim} \operatorname{Hom}_{C_{D}}\left(\mathscr{O}_{D}, K\right)=1$. If $K$ is algebraically closed, it's equivalent to any of the following: the identity element $(1, \ldots, 1) \in \mathscr{O}_{C}$ is in $\mathscr{C}_{D}$, but no other idempotent; every element $\left(f_{1}, \ldots, f_{r}\right) \in m_{D}$ has each $f_{E}$ in the maximal ideal $t_{E} A_{E}$ of $A_{E}$; every nonunit of $\mathscr{C}_{D}$ is nilpotent.

(c) If $Y \rightarrow X$ is a normalisation localised in codimension 1 as at the start of $\S 3$, the conductor subschemes $C \subset Y$ and $D \subset X$ define a part-filling $\mathscr{O}_{D} \subset \mathscr{O}_{C}$, with (ii) the definition of the conductor; (iii) will be equivalent to $X$ Gorenstein. (iii) obviously implies (ii) and also $\ell\left(\mathscr{O}_{D}\right)=\ell\left(\mathscr{O}_{C} / \mathscr{O}_{D}\right)$. The main point to prove in Theorem 3.2 is that

$$
\text { (ii) } \Rightarrow \ell\left(\mathscr{O}_{D}\right) \leq \ell\left(\mathscr{O}_{C} / \mathscr{O}_{D}\right) \text {, and equality } \Rightarrow \text { (iii). }
$$

(d) Every part-filling arises as a conductor subscheme for a normalisation $\pi: Y \rightarrow X$ of a reduced Noetherian local scheme $X$ : just take $X$ and $Y$ to be Spec of

$$
\mathscr{O}_{Y}=\prod R_{E} \text { and } \mathscr{\mathscr { O }}_{X}=\left\{f \in \mathscr{O}_{Y} \mid f \mapsto \bar{f} \in \mathscr{O}_{D} \subset \mathscr{O}_{C}\right\} .
$$

In the purely geometric case, when $\mathscr{O}_{D}$ is a $K$-algebra and $K=K_{E}$, you can even assume that each component of $Y$ is $\cong \mathbf{A}_{K}^{1}$, but the more general category also allows amusing things like reduced divisors on an arithmetic surface with some components in fibres (characteristic $p$ ) and some horizontal (mixed characteristic). So scheme theory suggests, for example, glueing together a ring of integers in a number field and a projective curve over a finite field, constructions which a number theorist may not immediately think of as natural.

(e) I've forgotten what I wanted to say here. Oh yes, the definition could in principle be generalised by allowing $K$ and the $K_{E}$ to be finite extensions of a common subfield $k_{0}$, but the extra generality is illusory, e.g., by [Matsumura, Theorem 28.3, (ii)] in the equal characteristic case.

(f) At cherry blossom time in Kyoto, in connection with the inequality in (II), I conjectured foolishly that an Artinian ring $A$ having a faithful module $M$ of finite length should satisfy $\ell(A) \leq \ell(M)$. A counterexample: the ring of $2 n \times 2 n$ matrixes having zero entries except in the top right $n \times n$ block, and equal diagonal entries, is clearly a commutative local $k$-algebra of length $n^{2}+1$ having $k^{2 n}$ as a faithful module.

3.6 I now proceed to prove Theorem 3.2 and other matters of interest in 5 easy steps. Each step consists of a statement for an abstract part-filling $\mathscr{O}_{D} \subset \mathscr{O}_{C}$, that makes sense without mention of $X$ and $Y$, then more-or-less trivial consequences for $Y \rightarrow X$.

Step 1. $\operatorname{ker} \operatorname{Tr}_{C / D}$ is the $\mathscr{O}_{D}$-dual of $\mathscr{O}_{C} / \mathscr{O}_{D}$, that is,

$$
\mathscr{O}_{C} / \mathscr{O}_{D} \stackrel{d}{-} \operatorname{ker}\left\{\operatorname{Tr}_{C / D}: \omega_{C} \rightarrow \omega_{D}\right\}=\omega_{X} / \omega_{Y}
$$


is just the restriction from $\mathscr{Q}_{C}$ to $\mathscr{O}_{D}$, that is, the dual of the inclusion map $\mathscr{O}_{D} \hookrightarrow \mathscr{O}_{C}$, so clearly

$$
\text { ker } \operatorname{Tr}=\operatorname{Hom}_{k_{0}}\left(\mathscr{G}_{C} / \mathscr{G}_{D}, k_{0}\right) \text {. }
$$

The general case is exactly the same on replacing $\operatorname{Hom}_{k_{0}}\left(-, k_{0}\right)$ by $\operatorname{Hom}_{A_{D}}\left(-, \omega_{D}\right)$ throughout.

Q.E.D.

Step 2. Therefore $\ell\left(\mathscr{O}_{C} / \mathscr{O}_{D}\right)=\ell\left(\operatorname{ker} \operatorname{Tr}_{C / D}\right)=\ell\left(\omega_{X} / \omega_{Y}\right)$ and the $\mathscr{C}_{D}$-module ker $\operatorname{Tr}_{C / D}$ is faithful, that is, $\operatorname{Ann}_{C_{D}}\left(\operatorname{ker} \operatorname{Tr}_{C / D}\right)=0$; hence

$$
\mathscr{C}=\operatorname{Ann}_{\epsilon_{X}}\left(\mathscr{O}_{Y} / \mathscr{O}_{X}\right)=\operatorname{Ann}_{c_{X}}\left(\omega_{X} / \omega_{Y}\right) \text {. }
$$

Proof. This follows from Step 1, since the dual $\mathscr{O}_{D}$-modules $\mathscr{O}_{C} / \mathscr{O}_{D}$ and $\operatorname{ker} \operatorname{Tr}_{C / D}$ have the same length and the same annihilator; and the $\mathscr{Q}_{X}$-module structures come from $\mathscr{O}_{X} \longrightarrow \mathscr{O}_{D}$.

Q.E.D

Step 3. For each $E$, write $t_{E}^{\prime}=\left(1, \ldots, t_{E}, \ldots, 1\right) \in \mathscr{O}_{C}$; this corresponds to a function on $Y$ with zero of order 1 along $E$, and no zeros along other components. Then for each E, I claim that

$$
\text { ker } \operatorname{Tr}_{C / D} \not \subset t_{E}^{\prime} \cdot \omega_{C} \text {, }
$$

that is, $\operatorname{ker} \operatorname{Tr}_{C / D}$ contains a basis of the localisation $\omega_{C, E} \cong \mathscr{C}_{C . E}=A_{E}$.

Thus $\omega_{X} \subset \omega_{Y}(C)$ contains a local basis of $\omega_{Y}(C)$ at the generic point of each $E$, that is, an element of $\omega_{Y}(C)$ with pole of order exactly $n_{E}$. Therefore $\omega_{Y}(C)$ is the $S_{2}$ isation of $\mathscr{O}_{Y} \cdot \omega_{X}$; and $\omega_{Y}$ the $S_{2}$ isation of $\mathscr{C} \cdot \omega_{X}$, so the hypothesis on $X$ can be omitted in the conclusion of Proposition 2.3. (Note that the last two statements are for a general variety before localising, but only the local statement in codimension 1 requires proof.)

Proof. Consider the element $s_{E}^{\prime}=\left(0, \ldots, t_{E}^{n_{E}-1}, \ldots, 0\right) \in \mathscr{O}_{C}$. Assuming by contradiction that $\operatorname{ker} \operatorname{Tr}_{C / D} \subset t_{E}^{\prime} \cdot \omega_{C}$, I prove that

$$
0 \neq s_{E}^{\prime} \in \operatorname{Ann}_{{ }^{\prime} D}\left(\operatorname{ker} \operatorname{Tr}_{C / D}\right) \text {, }
$$

which contradicts Step 2. To do this, I must prove both that multiplication by $s_{E}^{\prime}$ is zero, and that $s_{E}^{\prime} \in \mathscr{O}_{D}$. Obviously $0 \neq s_{E}^{\prime} \in \mathscr{C}_{C}$, and the multiplication map $\mu_{s_{E}}: \mathscr{O}_{C} \rightarrow \mathscr{O}_{C}$ is $\mathscr{O}_{C}$-linear, therefore $\mathscr{C}_{D}$-linear. The dual $\mathscr{C}_{D}$-linear map $\omega_{C} \rightarrow \omega_{C}$ induced by $\mu_{s_{E}^{\prime}}$ kills $t_{E}^{\prime} \cdot \omega_{C}$, and therefore by the assumption also ker $\operatorname{Tr}_{C I D}=\operatorname{Hom}_{\epsilon_{D}}\left(\mathscr{O}_{C} / \mathscr{O}_{D}, \omega_{D}\right)$. Finally, by duality it follows that $\mu_{S_{E}^{\prime}}(\overbrace{C})$ $=\mathscr{O}_{C} \cdot s_{E}^{\prime} \subset \mathscr{O}_{D}$, so that in particular $s_{E}^{\prime} \in \mathscr{C}_{D}$. This is the required contradiction.

The remaining assertions follow easily.

Q.E.D. 
Step 4. $\ell\left(\mathscr{O}_{D}\right) \leq \ell\left(\operatorname{ker} \operatorname{Tr}_{C / D}\right)=\ell\left(\mathscr{O}_{C} / \mathscr{O}_{D}\right)=\ell\left(\omega_{X} / \omega_{Y}\right)$; and if the residue field has cardinality

card $K>$ number of components $E$ of $C$, in particular if $K$ is infinite, then there exists an inclusion $\mathscr{O}_{D} \hookrightarrow \operatorname{ker} \operatorname{Tr}_{C / D}=\omega_{X} / \omega_{Y}$.

Proof. For each $E$, I can pick an element $s_{E} \in \operatorname{ker} \operatorname{Tr}_{C / D}$ which is a local basis of $\omega_{C}$ at the generic point of $E$ by Step 3. Under the given condition on the cardinality of $K$, it's easy to see that a suitable linear combination $s=\sum f_{E} s_{E}$ with $f_{E} \in \mathscr{O}_{D}$ is a local basis of $\omega_{C}$ at the generic point of every $E$; therefore $f \mapsto f \cdot s \in \omega_{C}$ is an injective map $\mathscr{O}_{C} \rightarrow \omega_{C}$ (in fact an isomorphism), so its restriction to $\mathscr{O}_{D}$ is also injective.

This proves the inequality $\ell\left(\mathscr{O}_{D}\right) \leq \ell\left(\mathscr{O}_{C} / \mathscr{O}_{D}\right)=\ell\left(\omega_{X} / \omega_{Y}\right)$ in the case that the residue field $K$ is infinite. For small $K$, the inequality (but not the inclusion) reduces without difficulty to the case of infinite $K$ by the standard trick [Matsumura, p. 114] of passing to the flat overring $\mathscr{O}_{D} \mapsto \mathscr{O}_{D}(x)$, which makes the purely transcendental extension $K \mapsto K(x)$ of the residue field.

Q.E.D.

Step 5. The equality $\ell\left(\mathscr{O}_{D}\right)=\ell\left(\operatorname{ker} \operatorname{Tr}_{C / D}\right)=\ell\left(\omega_{X} / \omega_{Y}\right)$ (or $n=2 \delta$ in [Serre, Ch. IV,$\S 11])$ is a necessary and sufficient condition for $\mathscr{O}_{D}$ to be a half-filling of $\mathscr{O}_{C}$, or for $X$ to be Gorenstein in codimension 1; dedicated algebraists can find a further halfdozen equivalent conditions in terms of primary decomposition, socles and homological algebra.

Proof. ker $\operatorname{Tr}_{C / D} \stackrel{\mathrm{d}}{\mathscr{O}_{C}} / \mathscr{O}_{D}$ by Step 1, so (iii) in Definition 3.4 implies that $\ell\left(\mathscr{O}_{D}\right)=\ell\left(\operatorname{ker} \operatorname{Tr}_{C / D}\right)$. Conversely, if the lengths are equal, the inclusion $\mathscr{O}_{D} \hookrightarrow \operatorname{ker} \operatorname{Tr}_{C / D}$ of Step 4 must be an isomorphism, which proves the result if $K$ is big enough. As before (Corollary 2.8, Proof of (ii) $\Rightarrow$ (i)), to deal with the case of finite residue field $K$ one has to make a finite faithfully flat extension $\mathscr{O}_{X}$ inducing a sufficiently big residue field extension, and argue on a finite module over a local ring which becomes free after a faithfully flat extension. The last sentence is obvious.

Q.E.D.

3.7 The simplest application of these ideas is the description of the codimension 1 behaviour of nonnormal del Pezzo surfaces stated in the introduction. Everything is still localised at a codimension 1 point of $X$.

Theorem. (I) Let $\mathscr{O}_{C}=\prod R_{E} /\left(t_{E}{ }^{n_{E}}\right)$, and assume that $n_{E}=1$ for some E. If $\mathscr{O}_{C}$ has a half-filling $\mathscr{O}_{D}$, then $\mathscr{O}_{D}=k(\Gamma)=K$ is a field (where $\Gamma=D_{\text {red }}$ ), and $\mathscr{O}_{C}$ is either $\cong K \times K$, or a quadratic extension field of $K$.

That is, if $X$ is nonnormal and Gorenstein, and $C \subset Y$ has a reduced component $E$, then $X$ has ordinary double points in codimension 1 along $\Gamma$. In characteristic 2 , this includes inseparable ordinary double points, that is, the quadratic extension may be an 
inseparable cover $E \rightarrow \Gamma$, in which case every geometric transverse section of the singularity of $X$ along $\Gamma$ is a cusp; compare 1.3,(A).

(II) Assume that $\mathscr{O}_{C}=\prod R_{E} /\left(t_{E}{ }^{2}\right)$, with all the $n_{E}=2$, and that $R_{E} /\left(t_{E}\right)$ $=k(E)=K$ for each $E$; write $T_{E}^{*}=\left(t_{E}\right) /\left(t_{E}{ }^{2}\right) \subset R_{E} /\left(t_{E}{ }^{2}\right)$ for the cotangent space of $R_{E}, a$ 1-dimensional $K$-vector space. Let $\mathscr{O}_{D} \subset \mathscr{O}_{C}$ be a half-filling. Then $m_{D} \subset \sum T_{E}^{\prime}$ is a $K$-vector subspace of codimension 1 , and if there are $\geq 2$ components $E$, it involves every summand:

$$
m_{D} \not \subset \sum_{E^{\prime} \neq E} T_{E^{\prime}}^{+}
$$

That is (for varieties over a field, for simplicity), suppose that $X$ is nonnormal and Gorenstein, and that every component $E$ has multiplicity 2 in $C$, and maps birationally to $\Gamma=D_{\text {red }} \subset \operatorname{Sing} X$. Then the transverse singularity of X along $\Gamma$ is a cusp $\left(y^{2}=x^{3}\right)$ if $r=1$, a tacnode $\left(y^{2}=x^{2} y\right)$ if $r=2$, and $r$ concurrent lines in $\mathbf{A}^{(r-1)}$ with no $(r-1)$ in a hyperplane for $r \geq 3$.

In (II), I assume that the residue field extensions are trivial $R_{E} / t_{E}=k(E)=K$ mainly out of spinelessness; this case is sufficient for my del Pezzo surfaces, since by Theorem 1.1, the only multiple locuses in $C$ are double lines. More generally, $T_{E}=\left(t_{E}\right) /\left(t_{E}{ }^{2}\right) \cong k(E)$ and $m_{D}=\operatorname{ker} \psi$, where $\psi: \sum T_{E} \rightarrow K$ is a nonzero multiple of the trace map $\operatorname{Tr}_{k(E) / K}$ on each piece.

3.8 Proof. (I) The notation $\mathscr{O}_{C}=\prod A_{E}$ with $A_{E}=R_{E} /\left(t_{E}{ }^{n_{E}}\right)$ is as above; since $X$ is nonnormal, $\sum n_{E} \cdot[k(E): K] \geq 2$, so that either there's more than one component $E$, or a component with $n_{E} \geq 2$ or $[k(E): K] \geq 2$.

The dual statement to the local condition $\operatorname{dim} \operatorname{Hom}_{{ }_{D}}\left(\mathscr{O}_{D}, K\right)=1$ on $\mathscr{C}_{D}$ (see 3.5, (b)) is that its socle

$$
\left\{s \in \omega_{D} \mid m_{D} \cdot s=0\right\}=\operatorname{Hom}_{{ }_{D}}\left(K, \omega_{D}\right)
$$

is 1-dimensional over $K$. Assuming $\mathscr{O}_{D} \subset \mathscr{O}_{C}$ is a half-filling, $\omega_{D}=\mathscr{O}_{C} / \mathscr{C}_{D}$.

If a component $E$ of $C$ has $n_{E}=1$ then $A_{E}=k(E) \subset \mathscr{Q}_{C}$ is killed by $m_{D}$; hence $S=\Sigma_{n_{E}=1} k(E) \subset \mathscr{O}_{C}$ is a $K$-vector subspace of the socle of $\mathscr{C}_{C}$ (as an $\mathscr{O}_{D}$-module). Consider the projection of $S$ to $\mathscr{O}_{C} / \mathscr{O}_{D}$. The local condition means that

$$
\mathscr{O}_{D} \cap S \cong\left\{\begin{array}{l}
K \text { if there are no further components; } \\
0 \text { if there are components with } n_{E} \geq 2 .
\end{array}\right.
$$

Therefore, if there are no further components, $S$ contributes

$$
\sum_{n_{E}=1}[k(E): K]-1 \leq 1
$$

to the $K$-dimension of the socle, which leads at once to the conclusion (I). 
If there are components with $n_{E} \geq 2$, I look for a contradiction. First, $S$ contributes $\sum_{n_{E}=1}[k(E): K] \leq 1$ to the dimension of the socle. It follows that $S=K$, hence $\mathscr{Q}_{C}$ is of the form $K \times \mathscr{O}_{C^{\prime}}$, and by the local condition $\mathscr{O}_{D}$ doesn't meet the first factor, so projects isomorphically to $\mathscr{O}_{D} \subset \mathscr{O}_{C^{\prime}}$. But $\ell\left(\mathscr{O}_{D}\right)>\ell\left(\mathscr{O}_{C^{\prime}} / \mathscr{O}_{D}\right)$, so $\mathscr{O}_{D} \subset \mathscr{O}_{C^{\prime}}$ is not a part-filling by 3.6 , Step 4 ; thus $\mathscr{O}_{C^{\prime}} / \mathscr{O}_{D}$ is an unfaithful $\mathscr{O}_{D}$-module. If $n \in \mathscr{O}_{D}$ kills $\mathscr{O}_{C^{\prime}} / \mathscr{O}_{D}$ then there is a case division:

Subcase $n$ a unit. Then $\mathscr{O}_{C^{\prime}} / \mathscr{O}_{D}=0$, which gives $\mathscr{O}_{C} \cong K \times K$; this certainly happens, but not under the current case assumption $n_{E} \geq 2$.

Subcase $n \in m_{D}$. Then $n \cdot \mathscr{C}_{C^{\prime}} \subset \mathscr{O}_{D}$ and $n \cdot K=0$ implies at once that $n \cdot \mathscr{G}_{C}=n \cdot\left(K \times \mathscr{G}_{C^{\prime}}\right) \subset \mathscr{O}_{D}$, contradicting the assumption that $\mathscr{O}_{C} / \mathscr{O}_{D}$ is faithful.

(II) Suppose there are $r$ factors so that $\mathscr{O}_{C}=\prod R_{E} /\left(t_{E}{ }^{2}\right)$ has length $2 r$. Thus $\mathscr{O}_{D}$ has length $r$, and by the local condition, $\mathscr{O}_{D} \subset \mathscr{O}_{C}$ maps to the diagonal $K \subset \Pi k(E)$ with kernel $m_{D}=\mathscr{O}_{D} \cap \sum T_{E}$ of length $(r-1)$. If $r=1$ there's nothing more to prove.

The dual of the diagonal map of $\mathscr{O}_{D} \subset \mathscr{Q}_{C}$ to $K \subset \prod k(E)$ is the inclusion of the socle of $\omega_{D}=\mathscr{C}_{C} / \mathscr{O}_{D}$, which is a map $K \rightarrow\left(\sum T_{E}\right) / m_{D}$ with each component nonzero; by duality this is surjective, so that $m_{D} \subset \sum T_{E}$ is a codimension one subspace involving each summand.

The geometric statement about $Y \rightarrow X$ is easy to see. For example if $r=1$ then $C=2 E$, there is a morphism $\varphi: 2 E \rightarrow \Gamma$ which is birational when restricted to $E$, and $\mathscr{O}_{X} \subset \mathscr{O}_{Y}$ is the ring of functions $f$ such that $f_{\mid 2 E} \in \mathscr{O}_{\Gamma}$. That is, $Y$ is pinched along $\varphi$ to give a codimension 1 locus of cusps.

Q.E.D.

3.9 Derivations and case (III) of Theorem 3.7. I now show how a subring $\mathscr{O}_{D} \subset \mathscr{O}_{C}$ in case (II) of the Theorem 3.7 is specified in practical calculations. Let $\pi: Y \rightarrow X$ be a normalisation of a reduced $S_{2}$ scheme with $\varphi: C \rightarrow D$ the conductor locus. Suppose that $\Gamma=D_{\text {red }}$ is irreducible, and that above its generic point, $\pi: Y \rightarrow X$ falls under case (II) of Theorem 3.7.

The glueing map $\varphi: C \rightarrow D$ can be factored as a composite of two maps; let $D^{+}$ be the variety homeomorphic to $D$, but with $\mathscr{O}_{D^{+}} \subset \varphi_{1} \mathscr{O}_{C}$ defined by

$$
\mathscr{O}_{D^{+}}=\left\{\left\{h_{l}\right\} \in \varphi \mathscr{C}_{C} \mid \operatorname{im} h_{l} \in k(E)=K \text { is independent of } i\right\}
$$

That is, $C \rightarrow D^{+}$does only the set-theoretic glueing, leaving the $r$ tangent spaces generically transversal, whereas $D^{+} \rightarrow D$ squashes up a first order disc in $r$-space over $k(\Gamma)$ into a first order disc in $(r-1)$-space. Geometrically, $D^{+}$corresponds to the "transversalisation" of the nonnormal locus of $X$, that is, $Y \rightarrow X^{+} \rightarrow X$ where $X^{+}$has a multiple curve with $r$ generically transverse branches.

Although this is not very intuitive, it's important to understand that already on the generic point, $D^{+} \rightarrow D$ contains nontrivial information of a differential nature: for example, if $r=1$ then $C=D^{+}$, and $\varphi$ maps a normal field to $C_{\text {red }} \subset C$ to a vector field 
on $D$.

Proposition. Under the above assumptions, there exists a (rational) derivation

$$
\Delta: \mathscr{O}_{D^{+}} \rightarrow\left(\mathscr{O}_{D_{\text {red }}^{+}}\right)_{\text {gen }}=k(E)
$$

such that $\mathscr{O}_{D}=\operatorname{ker} \Delta \subset \mathscr{O}_{D^{+}}$.

See 4.4-5 for a much more concrete description in the particular case of interest.

Proof. Write $N_{1}$ and $N_{2}$ for the nilpotent kernels of $\mathscr{O}_{D^{+}} \rightarrow \mathscr{O}_{D_{\text {red }}^{+}}$and $\mathscr{O}_{D} \rightarrow \mathscr{O}_{D_{\text {red }}}$; this gives the exact diagram

$$
\begin{aligned}
& 0 \rightarrow N_{2} \rightarrow \mathscr{O}_{D} \quad \rightarrow \quad \mathscr{C}_{D_{\text {red }}} \rightarrow 0 \\
& \cap \cap \cap \cap \\
& \begin{array}{llllllllll}
0 & N_{1} & \rightarrow & \mathscr{O}_{D^{+}} \\
& \downarrow & & & \downarrow & \mathscr{C}_{D_{\text {red }}^{+}} & \rightarrow & 0
\end{array} \\
& 0 \rightarrow N_{1} / N_{2} \rightarrow \mathscr{O}_{D^{+}} / \mathscr{O}_{D} .
\end{aligned}
$$

Looking only at generic stalks, the assumptions in Theorem 3.7, (II) give that $\mathscr{O}_{D_{\text {red }}}=\mathscr{O}_{D_{\text {red }}^{+}}$, so that

$$
\left(N_{1} / N_{2}\right)_{\text {gen }}=\left(\mathscr{O}_{D^{+}} / \mathscr{O}_{D}\right)_{\text {gen }},
$$

and the left-hand side is a 1-dimensional vector space over $k(E)$.

Now (a) all the above maps are $\mathscr{O}_{D}$ linear; and (b) since $N_{1} \subset \mathscr{C}_{D^{+}}$is an ideal with square zero, all the sheaves $N_{1}, N_{2}$ and $N_{1} / N_{2}$ in the left-hand column have both $\mathscr{O}_{D^{+}}$-module and $\mathscr{O}_{D}$-module structures that are compatible. The fact that $\Delta: \mathscr{O}_{D^{+}} \rightarrow\left(\mathscr{O}_{D_{\text {red }}}\right)_{\text {gen }}$ is well defined and a derivation follows formally.

Q.E.D.

\section{§4. The Glueing Map $\varphi: C \rightarrow D$ and Proof of Theorem 1.5}

4.0 The starting point in this section is a disjoint union

$$
(C \subset Y)=\coprod\left(C_{\imath} \subset Y_{\imath}\right)
$$

of surfaces $C_{\imath} \subset Y_{t}$ taken from the list of Theorem 1.1 (all defined over the same algebraically closed field). I study the possibilities for glueing $Y$ by a morphism $\varphi: C \rightarrow D$ to get a connected Gorenstein surface $X$; since $\pi^{\prime} \omega_{X}=\omega_{Y}(C)=C_{Y}(-1)$, it will follow automatically that the invertible sheaf $\omega_{X}{ }^{-1}$ is ample, so $X$ is a del Pezzo surface. The main result Theorem 1.5 can be viewed as a classification of nonnormal del Pezzo surfaces: under extra conditions they are just the surfaces $X$ described in $1.3-4$.

A nice simplifying feature is that I hardly need to work with $X$ and $Y$ at all: given that $\omega_{Y}(C) \cong \mathscr{O}_{Y}(-1)$, it follows by Corollary 2.8 , iv that $X$ is Gorenstein if and only if 
$\operatorname{ker}\left\{\operatorname{Tr}_{C / D}: \varphi \cdot \omega_{C} \rightarrow \omega_{D}\right\}$ is an invertible $\mathscr{O}_{D}$-module. Technically, the main point is to determine the map $\operatorname{Tr}_{C / D}$, in order to verify the conditions on $s \in \varphi_{k} \omega_{C}$ that it (a) belongs to ker $\mathrm{Tr}$, and (b) is an $\mathscr{O}_{D}$-basis of ker $\mathrm{Tr}$.

4.1 The first approximation to Theorem 1.5 is a numerical treatment that considers only the degrees of the curve components of $\operatorname{Sing} X$ and $C$ with respect to the polarisations $\mathscr{O}_{X}(1), \mathscr{O}_{Y}(1)$, and the nature of $X$ at the generic point. Note that $\operatorname{deg} \Gamma=1$ or 2 doesn't imply automatically that $\Gamma$ is isomorphic to a line or conic, since a priori $\mathscr{O}_{X}(1)$ is not very ample.

Lemma. One of the following holds (compare 1.3-4):

(A) Sing $X=\Gamma$ is irreducible with $\operatorname{deg} \Gamma=1$, and $C=\pi^{-1} \Gamma \subset Y$ a nonsingular conic with $\operatorname{deg}(\varphi: C \rightarrow \Gamma)=2$; in this case $Y$ is irreducible, and $X$ has ordinary double points in codimension 1 along $\Gamma$. This includes the possibility of inseparable ordinary double points in characteristic 2 , see the note in Theorem 3.7, (I).

(B) Sing $X=\Gamma$ is irreducible with $\operatorname{deg} \Gamma=2$; then $Y$ has exactly 2 components $Y_{1}, Y_{2}$, the curves $C_{1} \subset Y_{1}$ and $C_{2} \subset Y_{2}$ are nonsingular conics mapping birationally to $\Gamma$, and $X$ has ordinary double points in codimension 1 along $\Gamma$.

$\left(\mathrm{C}_{1}\right)$ Sing $X=\Gamma$ is irreducible with $\operatorname{deg} \Gamma=1$ and $C=\pi^{-1} \Gamma \subset Y$ a line pair; in this case $Y$ is irreducible, and $X$ has ordinary double points in codimension 1 along $\Gamma$.

$\left(\mathrm{C}_{r}\right) X=\bigcup_{t=1}^{r} X_{t}$ is a cycle of $r \geq 2$ components $X_{t}$, where $X_{t}$ and $X_{t+1}$ meet generically transversally along a curve $\Gamma_{t}$ with $\operatorname{deg} \Gamma_{t}=1$ (the indexes are taken cyclically, that is, $X_{r+1}=X_{1}$ ). Each conductor locus $C_{\imath} \subset Y_{\imath}$ is a line pair $C_{l}=\ell_{l} \cup \ell_{l}^{\prime}$, whose two components map birationally to $\Gamma_{t-1}$ and $\Gamma_{\imath}$.

(D) Sing $X=\Gamma$ is irreducible with $\operatorname{deg} \Gamma=1$, and the conductor locus $C_{t} \subset Y_{t}$ in each component of $Y$ is a double line; in this case $X$ has the singularities in codimension 1 along $\Gamma$ described in Theorem 3.7,(II).

Proof. Each $C_{\iota}$ is a conic, so is either reduced or a double line. Above a component $\Gamma \subset \operatorname{Sing} X$, the behaviour of $Y \rightarrow X$ is described by Theorem 3.7, (I) if $\pi^{-1} \Gamma \subset C$ is reduced, or Theorem 3.7, (II) if it consists of double lines.

Because the glueing takes place in codimension 1 by Proposition 2.2, in order for $X$ to be connected, the components of $Y$ must be joined together along components of $C_{l}$. If $C$ has a nonsingular conic component, then $C \rightarrow \Gamma$ is either a double cover of a curve of degree 1, which implies that $Y$ is connected; or two conics covering a curve of degree 2, which implies that $Y$ has exactly 2 components. This gives cases (A-B). If say $C_{1}=\ell_{1} \cup \ell_{1}^{\prime} \subset Y_{1}$ is a line pair, then $\varphi: C \rightarrow D$ must glue $\ell_{1}^{\prime}$ birationally to some other line, say $\ell_{1}^{\prime \prime}=\ell_{2} \subset C_{2} \subset Y_{2}$, and so on, to form a cycle, giving case $\left(\mathrm{C}_{r}\right)$.

The remaining possibility is that $C \rightarrow D$ is made up of $r$ double lines mapping generically to $D \subset X$ as in Theorem 3.7 , (II).

Q.E.D. 
4.2 Proof of Theorem 1.5 for reduced $C$. Case (A) is very easy: the conductor $\mathscr{C} \subset \mathscr{O}_{X}$ defines the reduced curve $\Gamma$ at its generic point, hence everywhere. Therefore $D=\Gamma$. Since $C$ is normal, the form " $D$ normal under $C$ " of the $S_{2}$ condition discussed in Proposition 2.2 implies that $D$ is normal, so $D \cong \mathbf{P}^{1}$, and $\varphi: C \rightarrow D$ is isomorphic to a linear projection of a conic to a line.

Case (B) is similar: each of the two components $C_{1}, C_{2}$ maps birationally to $D=\Gamma$ under $\varphi$; so $S_{2}$ again implies that $D$ is normal, and the glueing just consists of identifying $C_{1}$ and $C_{2}$ by an isomorphism to $D$.

Case (C): as before, $\mathscr{C} \subset \mathscr{O}_{X}$ defines the reduced curve $D=\bigcup \Gamma_{l}$ at each generic point, hence everywhere, so that $D$ is reduced. According to Lemma 4.1, every component $\Gamma_{l}$ has exactly two line components $\ell_{l}^{\prime}$ and $\ell_{l}^{\prime \prime}$ of $C$ mapping birationally to it. At last I can use the Gorenstein condition of Corollary 2.8, iii to prove something nontrivial:

4.3 Claim. There is a unique vertex point $P \in D$ such that

$$
\varphi^{-1} P=\{\text { nodes of } C\},
$$

in other words, all the nodes of the $C_{l}$, and no other points, map to $P$.

The point is the following: $\varphi$ identifies two lines $\ell_{1}^{\prime}$ and $\ell_{l}^{\prime \prime}=\ell_{l+1}$ birationally to $\Gamma_{l}$, and each of these has a marked point $P_{l}^{\prime} \in \ell_{l}^{\prime}$ and $P_{l}^{\prime \prime} \in \ell_{l}^{\prime \prime}$, namely the nodes of $C_{l}=\ell_{\imath} \cup \ell_{\imath}^{\prime}$ and $C_{t+1}=\ell_{l+1} \cup \ell_{l+1}^{\prime}$. The claim is that these two points match up under the identification. Assuming this, it's clear from the $S_{2}$ condition that the $r$ components $\Gamma_{1}$ are all normal, and that they define $r$ linearly independent directions in the tangent space to $D$ at $P$. This implies Theorem 1.5 in case (C).

Proof of Claim 4.3. As pointed out in Remark 2.9, the trace map is birational in nature. The birational identifications of $\ell_{1}^{\prime}$ and $\ell_{1}^{\prime \prime}$ with $\Gamma_{1}$ under $\varphi$ identifies the 3 generic stalks of the dualising sheaves, $\omega_{\gamma, \text { gen }}, \omega_{/, \text {,gen }}$ and $\omega_{\Gamma, \text { gen }}$; here the subscript gen denotes the generic stalk, and I omit the secondary subscript $i$ from now on. The calculation of $\operatorname{Tr}_{C / D}$ is trivial, namely

$$
\omega_{\gamma^{\prime}, \mathrm{gen}} \oplus \omega_{\gamma_{\text {, gen }}} \ni\left(s^{\prime}, s^{\prime \prime}\right) \mapsto s^{\prime}+s^{\prime \prime} \in \omega_{\Gamma, \mathrm{gen}} .
$$

Thus $\left(s^{\prime}, s^{\prime \prime}\right) \in \operatorname{ker} \operatorname{Tr}$ if and only if $s^{\prime}=-s^{\prime \prime}$.

The birational identification of the nonsingular curves $\ell_{l}^{\prime}$ and $\ell_{l}^{\prime \prime}$ extends to a biregular identification. The elements $s^{\prime} \in \omega_{\prime^{\prime, g e n}}$ and $s^{\prime \prime} \in \omega_{\text {,"gen }}$ are rational sections of $\omega_{,}$, and $\omega_{, "}$, and if $s^{\prime}=-s^{\prime \prime}$ then obviously the zeros and poles of $s^{\prime}$ and $s^{\prime \prime}$ must match up under the identification. To get a contradiction, suppose that $Q^{\prime} \in \ell_{l}^{\prime}$ and $Q^{\prime \prime} \in \ell_{l}^{\prime \prime}$ are identified, where $Q^{\prime}=\ell_{l} \cap \ell_{i}^{\prime} \in C_{l}$ is the node and $Q^{\prime \prime} \in C_{t+1}$ a nonsingular point. The Gorenstein condition of Corollary 2.8 , iii is that ker $\operatorname{Tr}$ contains an $\mathscr{O}_{C}$-basis of $\omega_{C}$ at each point of $C$. I will deduce from this that ker $\operatorname{Tr}$ has a section $\left(s^{\prime}, s^{\prime \prime}\right)$ over 
the generic point of $\Gamma_{i}$ such that $s^{\prime \prime}$ is regular at $Q^{\prime \prime}$, but $s^{\prime}$ has a pole at $Q^{\prime}$, contradicting what I just said.

Let $\left(s^{\prime}, s^{\prime \prime}\right) \in \omega_{/, \text {gen }} \oplus \omega_{/ \text {, gen }}$ be a section of $\varphi_{*} \omega_{C}$ at the generic point of $\Gamma_{t}$. First, $Q^{\prime \prime}$ is not a node, so $\ell_{l}^{\prime \prime}=C$ near $Q^{\prime \prime}$, and in order for $\left(s^{\prime}, s^{\prime \prime}\right)$ to be in $\varphi_{*} \omega_{C}$ near $\varphi\left(Q^{\prime \prime}\right)$, clearly $s^{\prime \prime}$ must be regular at $Q^{\prime \prime}$. But on the other hand, if $Q^{\prime}=\ell_{l} \cap \ell_{l}^{\prime} \in C_{l}$ is the node of $C_{l}$, then an $\mathscr{O}_{C_{l}}$-basis of the stalk of $\omega_{C_{l}}$ at $Q^{\prime}$ is given by $\left(s, s^{\prime}\right)$ where $s \in \omega_{1}\left(Q^{\prime}\right)$ and $s^{\prime} \in \omega_{,},\left(Q^{\prime}\right)$ are bases; that is, $s^{\prime}$ must have a pole at $Q^{\prime}$. This completes the contradiction.

Q.E.D.

4.4 The nonreduced case. I first describe without proof the argument in the most important case $r=1$ so that the trusting or exhausted reader can skip the rest of the paper. By Lemma 4.1 , in this case $C$ is a double line $C \cong 2 \ell \subset \mathbb{P}^{2}$, and $\varphi: C \rightarrow D=\Gamma$ a morphism whose restriction to the line $\ell=C_{\text {red }}$ is birational. The $S_{2}$ condition that $\varphi_{*} \mathscr{O}_{C} / \mathscr{O}_{\Gamma}$ is torsion-free does not imply $\Gamma$ nonsingular.

An affine piece of $C$ is $\left(y^{2}=0\right) \subset \mathbb{A}_{x, y}^{2}$, so that the generic stalk of $\mathscr{O}_{C}$ is $k(x)[y] /\left(y^{2}\right)$, and $k(\Gamma)=k(x)$; the map $\varphi: C \rightarrow \Gamma$ at the generic point corresponds to an inclusion

$$
\begin{aligned}
& k(x) \hookrightarrow k(x)[y] /\left(y^{2}\right), \text { by } f \mapsto f-h_{\varphi} f^{\prime} y, \\
& \text { where } f^{\prime}=\frac{\mathrm{d} f}{\mathrm{~d} x} \text { and } h_{\varphi}=h_{\varphi}(x) \in k(x) ;
\end{aligned}
$$

$h=h_{\varphi}$ can be arbitrary. In other words, generically $\varphi$ is a "projection" of the double line $C=2 \ell$ back to the reduced line $\rho \stackrel{\text { bir }}{\sim} \Gamma$; and the projection of a "normal field" to $\ell \subset C$ defines a derivation $f \mapsto h f^{\prime}$ of $\mathscr{O}$, that is, a rational vector field on $\ell$.

In this situation, it's clear from the $S_{2}$ condition that $\ell \rightarrow \Gamma$ is one-to-one, and that $\mathscr{O}_{\Gamma} \subset \mathscr{O}_{C}$ is the sheaf of all functions of the form $f-h f^{\prime} y \in \mathscr{O}_{C}$, where both $f$ and $h f^{\prime}$ are regular on $\ell$; that is,

$$
\mathscr{O}_{\Gamma} \cong\left\{f \in \mathscr{O}, \mid h f^{\prime} \in \mathscr{O},\right\}
$$

thus $\mathscr{O}_{\Gamma} \mathrm{V} \mathscr{O}$, at the poles of $h$, so $\Gamma$ is nonnormal there. Clearly $\xi=x-h y$ is a rational section of $\mathscr{G}_{\Gamma}$ that bases the function field, $k(\Gamma)=k(\xi)$. One calculates (see 4.8) that in terms of the natural bases

$$
s_{C}=\operatorname{Res}_{\mathrm{A}^{2} / C}\left(\frac{\mathrm{d} x \wedge \mathrm{d} y}{y^{2}}\right) \in \omega_{C} \text { and } \mathrm{d} \xi \in \omega_{k(\Gamma)},
$$

the trace map $\operatorname{Tr}_{C / D}: \omega_{C} \rightarrow \omega_{D}$ at the generic point of $C$ is

$$
\operatorname{Tr}_{C / D}\left((f+g y) \cdot s_{C}\right)=\left(g+(h f)^{\prime}\right) \cdot \mathrm{d} \xi
$$

so that $(f+g y) \cdot s_{C} \in \operatorname{ker} \operatorname{Tr} \Leftrightarrow \mathrm{g}=-(h f)^{\prime}$.

For $(f+g y) \cdot s_{C}$ to be a basis of $\omega_{C}$ at a point $P \in C$ it's necessary and sufficient 
for $f, g$ to be regular and $f(P) \neq 0$. Now it's easy to see that at $P \in \ell$, there exists a unit $f \in \mathscr{O}$, with $(h f)^{\prime} \in \mathscr{O}$, if and only if either $h$ is regular at $P$, or chark $=p$ and $h$ has a pole at $P$ of order divisible by $p$ (compare the proof of Theorem 4.6). Using the Gorenstein criterion Corollary 2.8, iii, the results stated in the introduction follow from this: if chark $=0$ then $C \rightarrow D$ must be a linear projection of a conic to a line. But in characteristic $p$, the rational function $h$ is allowed to have poles of order $=n p$ for any $n \geq 1$; at such a pole $P \in \ell$, it's clear that the local ring is of the form

$$
\mathscr{C}_{\Gamma, P} \cong k\left[x^{i} \mid\{i \equiv 0 \bmod p \text {, or } i \geq p\}\right]_{(0)}
$$

(localised at $x=0$ ), so that $\Gamma=D$ has a "wild" cusp. Arbitrary poles of this form can happen, so $H^{1}\left(\mathscr{O}_{X}\right)$ can be arbitrarily large. Clearly the local ring of $\Gamma$ at $P$ needs $p$ generators $x^{p}$ and $x^{n p+1}, \ldots, x^{n p+p-1}$, so that this type of singularity cannot occur if $\Gamma$ is contained in a smooth 3-fold and $p \geq 5$ (or, more generally, if it's given that $\operatorname{dim} T_{P} X \leq \operatorname{char} k$ ). In fact it can be checked (see Exercise 4.12) that in the case when $Y$ is nonsingular,

$$
\begin{cases}\operatorname{dim} T_{P} X=\operatorname{dim} T_{P} \Gamma=p & \text { if } p \geq 3 \\ \operatorname{dim} T_{P} \Gamma=2, \operatorname{dim} T_{P} X=3 & \text { if } p=2 .\end{cases}
$$

Thus in this case, $X$ has hypersurface singularities if chark $=2$ or 3 .

4.5 In the general case, $C=\coprod_{l=1}^{r} C_{t}$ with each $C_{t}$ a double line $C_{l}=2 \ell_{t} \subset \mathbf{P}^{2}$. Restricting $\varphi$ defines a birational map $\varphi_{1}: \ell_{l} \rightarrow \Gamma=D_{\text {red }}=\operatorname{Sing} X$ on each of the reduced curves; I write $\ell$ for the normalisation of $\Gamma$, so that the $\varphi_{1}$ define isomorphisms $\ell_{l} \cong \ell$. Now, as in 3.9 , let $\varphi^{+}: C \rightarrow D^{+}$be the morphism that glues the $C_{t}$ to each other along this isomorphism. In other words, $D^{+}$is isomorphic to a first order infinitesimal neighbourhood of $\ell=\mathbf{P}^{1} \subset \mathbf{P}^{r+1}$, and $C_{1} \subset D^{+}$are double lines in $r$ transverse planes through $\ell$. Let $x$ be an affine parameter on $\zeta$ and $y_{t}$ a linear form in the plane of $C_{l}$ vanishing along $\ell_{1}$.

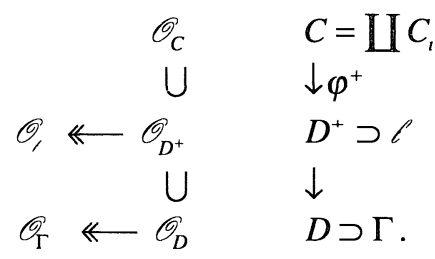

Obviously, the generic stalk of $\mathscr{O}_{D^{+}}$is $k(x)[y] /(\underline{y})^{2}$, where $\underline{y}$ is short for $y_{1}, \ldots, y_{r}$. By Proposition 3.9, $\mathscr{O}_{D}=\operatorname{ker} \Delta$ where $\Delta: \mathscr{C}_{D^{+}} \rightarrow k(x)$ is a rational derivation. But any derivation $\Delta: k(x)[\underline{y}] /(\underline{y})^{2} \rightarrow k(x)$ over $k$ is of the form

$$
\Delta(a, \underline{b}): f(x)+\sum g_{\imath}(x) y_{\imath} \mapsto a f^{\prime}+\sum b_{\imath} g_{\imath} \text { where } f^{\prime}=\frac{\mathrm{d} f}{\mathrm{~d} x},
$$

for some $a, b_{1}, \ldots, b_{r} \in k(x)$. Theorem 3.7, (II) contains the assertion that $b_{1} \neq 0$ for each $i$; the case $a=0$ is most welcome. A change of basis in $k(x)$ multiplies through 
$(a, \underline{b})$ by a nonzero element of $k(x)$.

4.6 I choose coordinates $x_{i}, y_{t}$ on an affine piece of each $C_{t}$, where $x_{i}, y_{i}$ are the pullback of the coordinates $x, y_{i}$ on $D^{+}$; a function on $C_{i}$ is of the form $f_{i}+g_{i} y_{i}$ where $f_{i}, g_{i}$ are rational functions of $x_{i}$, and from now on I usually omit mention of the substitution $x_{\imath} \mapsto x$ corresponding to the (nameless) identification of $\ell_{i}$ and $\ell$.

Theorem. $\operatorname{ker} \operatorname{Tr}_{C / D}$ contains a basis of $\varphi_{*} \omega_{C}$ at $P \in \ell$ (compare Corollary 2.8, iii) if and only if $b_{\imath} / b_{J}$ is a unit of $\mathscr{O}_{,, P}$ for all $i, j$, and either each $a / b_{\imath}$ is regular at $P$, or char $k=p$ and $a / b$, has a pole at $P$ of order divisible by $p$.

Proof. An element of $\varphi_{*} \omega_{C}$ is of the form

$$
s(\underline{f}, \underline{g})=\sum_{t=1}^{r}\left(f_{i}+g_{i} y_{l}\right) \cdot s_{l}, \quad \text { where } s_{i}=\operatorname{Res}_{\mathbf{A}^{2} / C_{l}} \frac{\mathrm{d} x_{l} \wedge \mathrm{d} y_{l}}{y_{i}^{2}}
$$

is a basis of $\omega_{C_{t}}$ over $\mathscr{O}_{C_{t}}$, and $\underline{f}=\left(f_{1}, \ldots, f_{r}\right), \underline{g}=\left(g_{1}, \ldots, g_{r}\right)$. The calculation of $\operatorname{Tr}_{C / D}$ given in 4.8 below proves that

$$
s(\underline{f}, \underline{g}) \in \operatorname{ker} \operatorname{Tr}_{C / D} \Leftrightarrow \frac{f_{l}}{b_{i}}=\frac{f_{1}}{b_{1}} \text { for all } i \geq 2 \text {, and }\left(\frac{a f_{1}}{b_{1}}\right)^{\prime}=-\sum_{i=1}^{r} g_{i} .
$$

Assuming (1) the result is easy: in order for $s(\underline{f}, \underline{g})$ to base $\omega_{C_{t}}$ at each $P_{t}$ lying over $P \in \ell$, each $f_{l}$ must be a unit, and $g_{i}$ regular. Therefore $b_{i} / b_{j}=f_{i} / f_{j}$ is a unit for all $i, j$. And I must be able to solve

$$
\left(\frac{a f_{1}}{b_{1}}\right)^{\prime}=-\sum g_{i} \text { with } g_{i} \text { regular and } f_{1} \text { a unit. }
$$

Suppose that $x$ is a local parameter at $P \in \ell$, and that $a / b_{1}=x^{m} \cdot u$ with $u$ a unit. It's obvious that regular functions $g_{i}$ can be chosen to solve (2) if and only if the left-hand side is regular. The left-hand side of (2) is the derivative of $f_{1} \cdot u \cdot x^{m}$, which up to multiplying by a nonzero constant is $m x^{m-1}+$ higher order terms. This is not regular if $m<0$ and $m \neq 0 \in k$, so that the condition on $a / b_{1}$ is necessary. When $m<0$, I can choose $f_{1}$ such that $f_{1}$ is a unit and $f_{1} \cdot u-1$ has a zero of order $\geq-m$ at $P$, so that

$$
\left(\frac{a f_{1}}{b_{1}}\right)^{\prime}=m x^{m-1}+\text { regular terms; }
$$

if also $m=0 \in k$ then this is regular, so I can solve (2) as before. This proves the theorem, assuming (1).

4.7 Tame. Using the notation of Theorem 4.6, $D_{\text {red }}$ is nonsingular if and only if $D_{\text {red }} \cong \ell=D_{\text {red }}^{+}$under each $P \in \ell$, which happens if and only if $a / b_{\imath}$ is regular, so that $x-\left(a / b_{l}\right) y_{\imath} \in \mathscr{O}_{D}$ is a local parameter. In local terms (also globally, see 4.11 ), if 
char $k=p$ then $a / b_{\imath}$ can have poles of order $=n p$ an arbitrary multiple of $p$; as discussed at the end of 4.4 , it's easy to see that in this case the local ring of $D_{\text {red }}$ is $k\left[x^{l} \mid\{i \equiv 0 \bmod p \text {, or } i \geq n p\}\right]_{P}$, so that it requires $p$ generators.

With $\pi: Y \rightarrow X$ and $\varphi: C \rightarrow D$ as in (0.2), I say that $\varphi: C \rightarrow D$ (or $X$ itself) is tame if either $C$ is reduced, or $C$ is nonreduced and $D_{\text {red }}$ nonsingular. Tameness is automatic under any of the following alternative extra assumptions on $X$ :

(i) The double locus $C \subset Y$ has a reduced component (see Theorem 3.7), (I)).

(ii) $\operatorname{char} k=0$.

(iii) chark $=p \geq 5$ and $X$ is locally a divisor in a nonsingular 3-fold, or, more generally, $\operatorname{char} k=p>\operatorname{dim} T_{P} X$ for all $P \in X$.

(iv) $C=\amalg C_{\imath}$ with each $C_{\imath}$ a double line and $\operatorname{Sing} X=D_{\text {red }} \cong \mathbf{P}^{1}$ (by definition).

(v) $\mathscr{O}_{X}(1)_{\mid D_{\text {red }}}$ is very ample (since then every component of $D_{\text {red }}$ is isomorphic to $\mathbf{P}^{1}$ or a plane conic).

(vi) $H^{1}\left(\mathscr{O}_{X}\right)=0$ or $\chi\left(\mathscr{O}_{X}\right)=1$ (see Corollary 4.10).

4.8 The calculation of $\operatorname{Tr}_{C / D}: \varphi_{*} \omega_{C} \rightarrow \omega_{D}$. The unpleasant thing is that $\operatorname{Tr}_{C / D}$ is $\mathscr{O}_{D}$-linear, but not $k(x)$-linear in general; to calculate it, I introduce new coordinates at the generic points of $C$ that are rational sections of $\mathscr{O}_{D} \subset \varphi_{*} \mathscr{O}_{C}$. The whole point is to write down sections of $\omega_{C}$ in terms of these new coordinates.

Recall that by Proposition 3.9 and 4.5, $\mathscr{O}_{D} \subset \mathscr{O}_{D^{+}}$is the subring defined by

$$
\mathscr{O}_{D}=\left\{f+\sum g_{l} y_{l} \in \mathscr{O}_{D^{+}} \mid a f^{\prime}+\sum b_{l} g_{l}=0\right\} \text {. }
$$

So in particular

$$
\begin{gathered}
\xi=x-\left(a / b_{1}\right) y_{1} \\
\text { and } \eta_{\imath}=y_{\imath}-\left(b_{\imath} / b_{1}\right) y_{1} \text { for } i=2, \ldots, r
\end{gathered}
$$

are rational sections of $\mathscr{O}_{D}$. I've chosen $\xi$ and $\eta_{l}$ so that they only mess up $C_{1}$, and not $C_{t}$ for $i=2, \ldots, r$ : the pullback of $\xi$ to $C_{1}$ is $\xi_{1}=x_{1}-\left(a / b_{1}\right) y_{1}$, and to $C_{t}$ is $\xi_{t}=x_{t}$ for $i \geq 2$; whereas each $\eta_{t}$ for $i \geq 2$ pulls back to $y_{t}$ on $C_{l}$, to $-\left(b_{t} / b_{1}\right) y_{1}$ on $C_{1}$ and to 0 on all components $C_{J}$ with $j \neq i$.

I have to translate

$$
s(\underline{f}, \underline{g})=\sum_{\imath=1}^{r}\left(f_{\imath}\left(x_{\imath}\right)+g_{\imath}\left(x_{t}\right) y_{\imath}\right) \cdot s_{\imath} \in \varphi_{+} \omega_{C}
$$

into the new coordinates. For this, first define new generic bases

$$
s_{\imath}^{\prime}=\operatorname{Res}_{\mathbf{A}^{2} / C_{l}} \frac{\mathrm{d} \xi_{\imath} \wedge \mathrm{d} y_{t}}{y_{l}{ }^{2}} \in \omega_{C_{l}} .
$$

There's not very much to calculate for the terms with $i \geq 2$, since $s_{l}=s_{\imath}^{\prime}$ and $f_{i}\left(x_{t}\right)+g_{l}\left(x_{t}\right) y_{i}=f_{l}\left(\xi_{l}\right)+g_{\imath}\left(\xi_{l}\right) y_{\imath}$. The fun comes when $i=1$; note that $\mathrm{d} x_{1}$ $=\left(1+\left(a / b_{1}\right)^{\prime} y_{1}\right) \cdot \mathrm{d} \xi_{1}+\left(a / b_{1}\right) \mathrm{d} y_{1}$. Thus 


$$
s_{1}=\operatorname{Res}_{\mathbf{A}^{2} / C_{1}} \frac{\mathrm{d} x_{1} \wedge \mathrm{d} y_{1}}{y_{1}^{2}}=\left(1+\left(a / b_{1}\right)^{\prime} y_{1}\right) \cdot s_{1}^{\prime} .
$$

Now using $y_{1}^{2}=0$ and the Taylor expansion for $f_{1}\left(x_{1}\right)=f_{1}\left(\xi_{1}+\left(a / b_{1}\right) y_{1}\right)$ gives

$$
\begin{aligned}
\left(f_{1}\left(x_{1}\right)+g_{1}\left(x_{1}\right) y_{1}\right) \cdot s_{1} & =\left(f_{1}\left(\xi_{1}\right)+\left(a / b_{1}\right) f^{\prime} y_{1}+g_{1} y_{1}\right) \cdot\left(1+\left(a / b_{1}\right)^{\prime} y_{1}\right) \cdot s_{1}^{\prime} \\
& =\left(f_{1}\left(\xi_{1}\right)+\left(a f / b_{1}\right)^{\prime} y_{1}+g_{1} y_{1}\right) \cdot s_{1}^{\prime} .
\end{aligned}
$$

Therefore,

$$
s(\underline{f}, \underline{g})=\left(\frac{a f}{b_{1}}\right)^{\prime} y_{1} \cdot s_{1}^{\prime}+\sum_{l=1}^{r}\left(f_{\imath}\left(\xi_{l}\right)+g_{l} y_{l}\right) \cdot s_{l}^{\prime} .
$$

Proof of (1). Since $\xi$ is a rational section of $\mathscr{O}_{D}$, the trace $\operatorname{Tr}_{C / D}$ on generic sections is $k(\xi)$-linear. Hence I view the generic stalk of $\varphi_{*} \omega_{C}$ as $\operatorname{Hom}_{k(\xi)}\left(\varphi_{*} \mathscr{O}_{C}, k(\xi)\right)$. The trace is just the restriction to $\mathscr{O}_{D}$ of elements of $\operatorname{Hom}_{k(\xi)}\left(\varphi_{\mathrm{r}} \mathscr{O}_{C}, k(\xi)\right)$, so a generic section of $\varphi_{*} \omega_{C}$ is in the kernel of $\operatorname{Tr}_{C / D}$ if and only if it kills the elements $1 \in \mathscr{O}_{D}$ and $\eta_{i} \in \mathscr{O}_{D}$ for $i \geq 2$, that form a $k(\xi)$-basis of $\left(\mathscr{O}_{D}\right)_{\text {gen }}$.

Now making the usual identification of $\omega_{C_{l}}$ with differentials, I get that the element $\left(f_{t}\left(\xi_{i}\right)+g_{\imath}\left(\xi_{i}\right) y_{i}\right) \cdot s_{\imath}^{\prime}$ is the function on $\mathscr{O}_{C_{l}}$ taking $a\left(\xi_{i}\right)+b\left(\xi_{l}\right) y_{i} \in \mathscr{O}_{C_{l}}$ into the residue of the product, that is, into the coefficient $a g_{l}+b f_{\imath} \in k(\xi)$ of $y_{t}$. Now using (3), it's easy to see that evaluating $s(\underline{f}, \underline{g})$ on $1_{\kappa_{D}}=\{1, \ldots, 1\} \in \varphi_{,} \mathscr{O}_{C}$ and $\eta_{i}$ $=y_{i}-\left(b_{t} / b_{1}\right) y_{1}$ gives

$$
\left(\frac{a f}{b_{1}}\right)^{\prime}+\sum_{\imath=1}^{r} g_{\imath} \quad \text { and } \quad f_{i}-\left(b_{\imath} / b_{1}\right) f_{1}
$$

The conditions for $\operatorname{Tr}_{C / D} s(\underline{f}, \underline{g})=0$ are obtained by setting both of these to 0 . This proves (1) and completes the proof of Theorem 4.6.

Q.E.D.

4.9 Proposition. Write $\ell, \mathscr{O}$, (1) for $\mathbb{P}^{1}$. Then in the notation of 4.5 , the structure sheaf of $D^{+}$is isomorphic to $\mathscr{O}_{D^{+}} \cong \mathscr{G}, \oplus \oplus_{l=1}^{r} \mathscr{O},(-1)$ as a sheaf of rings, with the second summand $N_{1}$ an ideal of square zero.

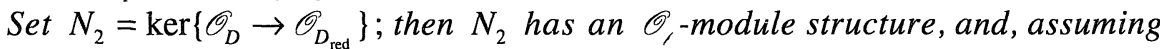
that $X$ is Gorenstein, $N_{2} \cong \oplus_{l=1}^{r-1} \mathscr{O},(-1)$. Suppose in addition that $X$ is tame (see 4.7). Then as a sheaf of rings $\mathscr{O}_{D} \cong \mathscr{O}, \oplus \oplus_{l=1}^{r-1} \mathscr{O},(-1)$. In other words, $D, \mathscr{O}_{D}(1)$ is isomorphic to a first order neighbourhood of $\mathbb{P}^{1}$ in $\mathbb{P}^{r}$, with the morphism $\varphi: \amalg C_{l} \rightarrow D$ linear on each component.

Proof. The first part is easy, since $\mathscr{O}_{D^{+}}$fits in the locally split exact sequence $0 \rightarrow N_{1} \rightarrow \mathscr{O}_{D^{+}} \rightarrow \mathscr{O}, \rightarrow 0$, with $N_{1}=\oplus_{l=1}^{r} \mathscr{Q},(-1)$, any two local splittings $s_{\imath}$ and $s_{J}$ 
differ by a derivation $\delta_{i, j}=s_{l}-s_{j}: \mathscr{O}, \rightarrow N_{1}$, and $H^{1}\left(\mathscr{D e r}\left(\mathscr{O}, N_{1}\right)\right)=H^{1}\left(\mathscr{H a m}\left(\Omega^{1}, N_{1}\right)\right)$ $=0$.

The $\mathscr{O}_{D^{+}}$or $\mathscr{C}$,-module structure of $N_{2}$ comes from the fact that $N_{1}$ is nilpotent of square 0 . Consider the projection $N_{1} \rightarrow N_{1} / N_{2}$. By the $S_{2}$ condition $N_{1} / N_{2}$ is a line bundle over $\ell$, and $N_{1} \rightarrow N_{1} / N_{2}$ is the map defined by the $b_{1}$. Since by Theorem 4.6 the $b_{l} / b_{J}$ are units, it follows that each direct summand $\mathscr{O}_{,}(-1)$ of $N_{1}$ (corresponding to the $y_{t}$ ) maps isomorphically to $N_{1} / N_{2}$, so that $N_{1} / N_{2} \cong \mathscr{O},(-1)$. Thus the kernel $N_{2}$ is also a direct sum of copies of $\mathscr{O},(-1)$.

In the notation of Theorem 4.6 , if any of the $a / b$, is a unit, then I can choose $\xi=x-b_{l} y_{1} \in \mathscr{O}_{D} \subset \mathscr{O}_{D^{+}}$which maps to a local parameter of $\mathscr{O}_{\text {; }}$; since I'm assuming this holds for all $P$, the exact diagram in 3.9 becomes

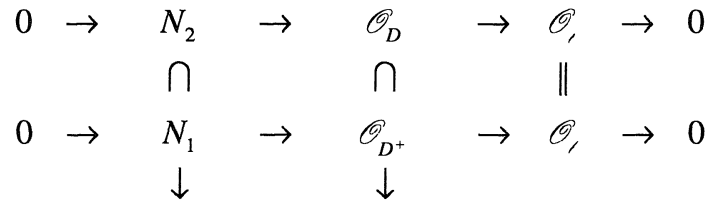

$$
\begin{aligned}
& N_{1} / N_{2}=\mathscr{O}_{D^{+}} / \mathscr{O}_{D} .
\end{aligned}
$$

The splitting of $\mathscr{O}_{D}$ as a sheaf of rings follows as before.

Q.E.D.

4.10 Corollary. (I) Let $X$ be a nonnormal del Pezzo surface. Then

$X$ is tame $\Leftrightarrow \chi\left(\mathscr{O}_{X}\right)=1 \Leftrightarrow H^{1}\left(\mathscr{C}_{X}\right)=0$;

and if this holds, $H^{1}\left(\mathscr{O}_{X}(n)\right)=0$ for all $n$.

(II) Assume that $X$ is tame and write $n=\operatorname{deg}_{\epsilon_{X}(1)} X=\left(\mathscr{O}_{X}(1)\right)^{2}$. Then $\mathscr{O}_{X}(1)$ is very ample if $n \geq 3$, generated by its $H^{0}$ if $n=2$, and has a single transverse base point if $n$ $=1$.

Proof of $(\mathrm{I})$. Obviously $h^{0}\left(\mathscr{O}_{X}\right)=1$ and $h^{2}\left(\mathscr{C}_{X}\right)=h^{0}\left(\mathscr{O}_{X}(-1)\right)=0$, and therefore in any case $\chi\left(\mathscr{O}_{X}\right)=1-h^{1}\left(\mathscr{O}_{X}\right)$. Also $\pi_{i} \mathscr{O}_{Y} / \mathscr{O}_{X}=\varphi_{i} \mathscr{O}_{C} / \mathscr{O}_{D}$ which gives

$$
\chi\left(\mathscr{O}_{Y}\right)-\chi\left(\mathscr{O}_{X}\right)=\chi\left(\mathscr{O}_{C}\right)-\chi\left(\mathscr{O}_{D}\right)
$$

now each $C_{l}$ is a plane conic, so that $\chi\left(\mathscr{O}_{Y_{1}}\right)=\chi\left(\mathscr{O}_{C_{1}}\right)=1$ for each $C_{l} \subset Y_{1}$, and hence $\chi\left(\mathscr{C}_{X}\right)=\chi\left(\mathscr{O}_{D}\right)$. But the conclusion of 4.2-3 and Proposition 4.9 is that in the tame case $D$ is either a line, a conic, a union of linearly independent lines through a point, or a first order neighbourhood of $\mathbf{P}^{1}$ in $\mathbf{P}^{r}$. These all have $\chi\left(\mathscr{O}_{D}\right)=1$; for example, in the last case, $\chi\left(\mathscr{O}_{D}\right)=\chi\left(\mathscr{O}_{\mathrm{p},}\right)=1$ follows from Proposition 4.9 .

$N_{2}=(r-1) \mathscr{O}(-1)$ by Proposition 4.9 , so that $\chi\left(N_{2}\right)=0$, and hence $\chi\left(\mathscr{O}_{X}\right)=\chi\left(\mathscr{O}_{D}\right)=\chi\left(\mathscr{O}_{D_{\text {red }}}\right)$. But if $\mathscr{O}_{D_{\text {red }}} \mathrm{V} \mathscr{O}$, then $\chi\left(\mathscr{O}_{D_{\text {red }}}\right) \leq 0$. Thus tame is equivalent to $\chi\left(\mathscr{O}_{X}\right)=1$. 
The rest of (I) is easy: using $\mathscr{O}_{D}=\mathscr{O}_{\zeta} \oplus(r-1) \mathscr{O}_{,}(-1)$ together with easy arguments on the $C_{i} \subset Y_{t}$ implies that both the maps $H^{0}\left(Y, \mathscr{O}_{Y}(n)\right) \longrightarrow H^{0}\left(C, \mathscr{O}_{C}(n)\right) \longrightarrow$ $H^{0}\left(D, \varphi_{*} \mathscr{O}_{C} / \mathscr{O}_{D}(n)\right)$ are surjective for $n>0$, so that $H^{1}\left(\mathscr{O}_{X}(n)\right)=0$ follows from the short exact sequence $0 \rightarrow \mathscr{O}_{X}(n) \rightarrow \mathscr{O}_{Y}(n) \rightarrow \varphi_{*} \mathscr{O}_{C} / \mathscr{O}_{D}(n) \rightarrow 0$. For $n<0$, just use duality.

Proof of (II). First, a similar argument to that just given shows that $H^{0}\left(\mathscr{O}_{X}(1)\right) \longrightarrow H^{0}\left(\mathscr{O}_{D}(1)\right)$; since $\mathscr{O}_{D}(1)$ is obviously very ample, sections $\mathscr{O}_{X}(1)$ embed $D$. Next, if $X$ is reducible, $H^{0}\left(\mathscr{O}_{X}(1)\right)$ embeds every component of $Y$. For either $Y_{l}$ is a plane, and $H^{0}\left(\mathscr{O}_{X}(1)\right)$ embeds $C_{\imath} \subset D$, hence also $Y_{i}$. Or $Y_{\imath}$ is not a plane, in which case $I_{C_{t}} \cdot \mathscr{O}_{Y_{t}}(1)$ is generated by its $H^{0}$, which is a direct summand of $H^{0}\left(I_{D} \cdot \mathscr{O}_{X}(1)\right)$. Finally, if $X$ is irreducible, then $\pi: Y \rightarrow X$ composed with the rational map corresponding to $\mathscr{O}_{X}(1)$ is just the linear projection of $Y \subset \mathbf{P}^{n+1}$ from a point in the plane of the conic $C$ but not on $C$.

Q.E.D.

4.11 The wild case. Suppose $\operatorname{char} k=p$. Then in the diagram of 3.9, $N_{1} / N_{2} \cong \mathscr{O},(-1)$, but $\mathscr{O}_{D^{+}} / \mathscr{O}_{D}$ can be $\mathscr{O},(N p-1)$ for any $N>0$. For this, just take the derivation $\mathscr{O}_{D^{+}} \rightarrow k(x)$ given by $(f, g) \mapsto a f^{\prime}+\sum g_{i}$, where $a \in k(x)$ is a rational function having poles of order exactly $n_{j} p$ at any points $P_{j} \in \ell$ (and zeros anywhere outside the $P_{J}$ ). It's clear from the proof of Corollary 4.10 that the corresponding surface $X$ will have $h^{1}\left(\mathscr{O}_{X}\right)=N(p-1)$, so $\chi\left(\mathscr{O}_{X}\right)=1-N(p-1)$. Shepherd-Barron kindly points out that the Picard scheme $\mathrm{Pic}^{0} X$ of $X$ is simply $N(p-1)$ copies of the additive group scheme $\mathbf{G}_{a}$ : in fact by deformation theory, $H^{2}\left(\mathscr{O}_{X}\right)=0$ implies that $\operatorname{Pic}^{0} X$ is reduced of dimension $h^{1}\left(\mathscr{O}_{X}\right)$; and it can't contain $\mathbf{G}_{m}$ or an Abelian variety, since then $X$ would have cyclic etale covers of arbitrarily large order, which is absurd in view of the concrete description of $X$ (in the irreducible case, $X$ is homeomorphic in the etale topology to its normalisation, a rational surface).

4.12 Exercise. (i) Suppose that chark $=p \geq 3$, and that $Y$ is smooth with affine coordinates $(x, y)$, such that $C:\left(y^{2}=0\right)$. In the notation of 4.4 , let $h=x^{-n p} \cdot h_{0}$ with $h_{0}=h_{0}(x)$ a unit and $n \geq 1$. Show that the local ring $\mathscr{O}_{X, P}$ is the localisation at 0 of

$$
k\left[u, v_{1}, \ldots, v_{p-1}\right], \text { where } u=x^{p} \text { and } v_{t}=x^{n p+i}-i h_{0} x^{t-1} y \text { for } i=1, \ldots, p-1 \text {. }
$$

[Hint: $\mathscr{O}_{X}$ consists of polynomials of the form $f(x)-h f^{\prime}(x) y+g(x, y) y^{2}$, where $f(x)$ and $h f^{\prime}(x)$ are regular, and $g(x, y)$ is arbitrary. Show that

$$
v_{\imath} v_{J}-u^{n} v_{i+J}=\text { unit } \times x^{i+J-2} y^{2},
$$

and find a similar trick giving the monomials $x^{l} y^{3}$.]

(ii) Take $p=3$ in (i). Show that

$$
\begin{gathered}
\mathbf{A}^{2}=Y \rightarrow X \subset \mathbf{A}^{3} \\
\text { given by }(x, y) \mapsto\left(x^{3}, x^{3 n+1}-h_{0} y, x^{3 n+2}-2 h_{0} x y\right)
\end{gathered}
$$


is the normalisation of the hypersurface $X:\left(u^{3 n+2}+u v_{1}^{3}+v_{2}^{3}=0\right) \subset \mathbf{A}^{3}$, a surface having cusps in codimension 1 along the parametrised curve $\left(t^{3}, t^{3 n+1}, t^{3 n+2}\right)$.

(iii) Similarly, if char $k=2$ then

$$
\begin{gathered}
\mathbf{A}^{2}=Y \rightarrow X \subset \mathbf{A}^{3} \\
\text { given by }(x, y) \mapsto\left(x^{2}, x^{2 n+1}+y, x y^{2}\right)
\end{gathered}
$$

is the normalisation of $X:\left(u\left(u^{2 n+1}+v^{2}\right)^{2}+w^{2}=0\right) \subset \mathbf{A}^{3}$, a surface with cusps along $\left(t^{2}, t^{2 n+1}, 0\right)$. This corresponds to $h=-x^{-2 n} \cdot h_{0}$ in 4.4 .

\section{References}

[Altman-Kleiman] Altman, A. and Kleiman, S., Introduction to Grothendieck duality, LNM 146.

[Artin] Artin, M., Algebraization of formal moduli: II. Existence of modifications, Ann. of Math., 91 (1970), 88-135.

[Bourbaki] Bourbaki, N., Algèbre commutative, Hermann, Paris.

[del Pezzo 1] del Pezzo, Pasquale, Sulle superficie dell'ordine $n$ immerse negli spazi di $n+1$ dimensioni, Rend. della R. Acc. delle Scienze Fis. e Mat. di Napoli, Sept. 1885.

[del Pezzo 2] - Sulle superficie dell' $n^{\text {no }}$ ordine immerse nello spazio di $n$ dimensioni, Rend. del circolo matematico di Palermo, 1 (1887), 241-271.

[Enriques] Enriques, Federigo, Le superficie algebriche , Zanichelli, Bologna, 1949.

[Grauert and Schneider] Grauert, H. and Schneider, M., Komplexe Unterräume und holomorphe Vektorbündel vom Rang zwei, Math. Ann., 230 (1977), 75-90.

[Grothendieck-Hakim] Grothendieck, A. (written up by M. Hakim), Modules et foncteurs dualisants, Exposé 4 of Cohomologie locale des faisceaux cohérents et théorèmes de Lefschetz locaux et globaux, (SGA 2), North-Holland, 1968.

[Grothendieck-Hartshorne] Grothendieck, A. (written up by R. Hartshorne), Local cohomology, LNM 41.

[Hartshorne] Hartshorne, R., Algebraic geometry, Springer.

[Horrocks] Horrocks, G., Birationally ruled surfaces without embeddings in regular schemes, Bull. London Math. Soc., 3 (1971), 57-60.

[Kollár] Kollár, J., Towards moduli of singular varieties, Comp. Math., 56 (1985), 369-398.

[Matsumura] Matsumura, H., Commutative ring theory, C.U.P., Cambridge, 1986.

[Mori] Mori, S., Three-folds whose canonical bundles are not numerically effective, Ann. of Math., (2) 116 (1982), 133-176.

[Mumford] Mumford, D., Appendix to Ch. 3 of Zariski, Algebraic surfaces, 2nd Ed., Springer, 1971.

[C3-f] Reid, M., Canonical singularities, in Journées de géometrie algébrique d'Angers, ed. A. Beauville, Sijthoff and Noordhoff, Alphen 1980, 273-310.

[YPG] - Young person's guide to canonical singularities, in Algebraic Geometry, Bowdoin 1985, Proc. of Symposia in Pure Math., $46: 1,345-414$, A.M.S., 1987.

[Reid] - Local aspects of duality, expository lecture, 12pp., approx. Nov. 1987.

[Semple and Roth] Semple, J. G. and Roth, L., Introduction to algebraic geometry, Oxford.

[Serre] Serre, J. -P., Groupes algébriques et corps de classes, Hermann, Paris, 1959 (English translation, Springer, 1990). 
